\title{
Breakage Characteristics of Granulated Food Products for Prediction of Attrition during Lean- Phase Pneumatic Conveying
}

\section{Authors}

Benjamin A. Kotzur(a), Michael S. A. Bradley(a), Robert J. Berry(a, b), Richard J. Farnish(a)

(a) The Wolfson Centre for Bulk Solids Handling Technology, University of Greenwich

(b) Corresponding author

Corresponding Author Details

Postal Address

The Wolfson Centre for Bulk Solids Handling Technology

University of Greenwich

Central Avenue

Chatham, Kent, ME4 4TB

Email: $\quad$ R.J.Berry@Greenwich.ac.uk

Telephone: + +44 (0)20 83318646 


\section{Abstract}

Pneumatic conveying is utilised in a variety of industries to convey food products exhibiting diverse handling characteristics. Attrition of particles caused by this conveying process can result in a number of undesirable outcomes such as loss in product quality or issues in subsequent handling processes. The ability to predict the breakage behaviour of particulate materials is desirable in both new system design and resolving issues in existing plants. This work considers two different particulate materials (Salt and Golden Breadcrumbs) across a range of particle sizes, and quantifies their breakage behaviour under varying impact conditions. Narrow size fractions of each material was degraded; material retained on $250 \mu \mathrm{m}$ and $355 \mu \mathrm{m}$

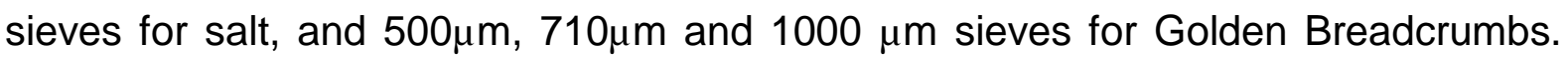
Velocity was found to be the most influential factor with respect to particle attrition. The results from the narrow size fraction tests were superimposed to form a simulated full size distribution breakage behaviour, which was then compared to the experimentally determined behaviour. A good agreement was found, however the proportion of material predicted for size fractions smaller than $355 \mu \mathrm{m}$ for Golden Breadcrumbs and $180 \mu \mathrm{m}$ for Salt was under-predicted. Recommendations for increasing accuracy of the prediction method are given.

\section{Keywords}

Particle attrition, Lean-Phase, Pneumatic Conveying 


\section{Introduction}

Pneumatic conveying is widely used in the food manufacturing industry to transport food products between process operations or to and from storage. These food products can take the form of powders or flake type bulk materials, including items such as tea leaves, granulated sugar, flour, and flavourings. The main advantages of using pneumatic conveying to transport materials throughout a process include product containment for hygiene or dust control, and the potential for flexibility in pipework routing in comparison to mechanical conveying. However, the fulfilment of the many benefits of pneumatic conveying can only be realised if the system is correctly configured and operated optimally. In instances where this is not the case, a common problem, is bulk material attrition due to excessive impact forces experienced by the particles as they pass through the system, and encounter changes in direction at bends. The final result can often be the generation of excess quantities of degraded particle size fractions - often in the form of fines or dust. The presence of these smaller particle sizes can have significant negative implications on; the reliability of subsequent process operations and the customer perceptions of product quality.

The research presented in this document will provide a comparative breakage analysis of two types of food products, one crystalline in structure, and the other non-crystalline. This information may then be used to predict with a greater degree of confidence, the magnitude of anticipated attrition when pneumatically conveying particulate materials.

There are three main modes of pneumatic conveying: 
1. Dense-phase describes conveying conditions when the entire cross-section of the pipe is occupied by particles at relatively low velocity (approximately $2-8 \mathrm{~m} / \mathrm{s})$.

2. Lean-phase describes conveying conditions when the particles are fully suspended in the air flow under high velocity conditions (greater than approximately $12 \mathrm{~m} / \mathrm{s})$.

3. Pulse/dune flow describes transient conveying conditions between Modes 1 and 2, and describes the flow of 'dunes' of material progressing through the pipeline.

This study will consider lean phase pneumatic conveying effects specifically, as the combination of high velocity and low particle concentration yields the most likely conditions to induce an increased level of particle attrition.

The term 'fines' in this research shall refer to the $D_{10}$ content of the virgin cumulative size distribution. Should the material within this size range make up too large a proportion of the overall mass, adverse material handling and performance issues can occur. These types of issues are extensive, and include:

1. Unreliable discharge from hoppers. An increase in the proportion of fine particles may inhibit particle flow through a hopper outlet through either arching or intermittent flow,

2. Dust generation, leading to explosion or health hazards through inhalation and skin contact,

3. Segregation of mixtures, whereby small particles become more concentrated in particular areas of a material bed.

4. Excess dust in commercial products leading to customer complaints, 
5. Error in volumetric dosing operations due to variations in bulk density caused by segregation and flow issues defined above.

The occurrence of such issues typically results in additional costs; both time and financial to the producers of the product. These costs are consumed by production down time amending the configuration or operating conditions of the existing process, or in reformulating the nature of the material handled.

This research seeks to provide an improved tool that can be applied to identify risk within processes. The approach will use predictive analysis of the product through empirical testing. Particle impact conditions can be carefully controlled within a centrifugal accelerator attrition tester, to provide comparative data for qualitative analysis. Then, based on a comparison with products previously handled, insight into the breakage behaviour may be attained and its subsequent attrition behaviour estimated.

A selection of advantages that predictive analysis would yield include:

1. Reduced requirement for post-installation troubleshooting,

2. Enhanced understanding of the operating parameters that cause an unacceptable degree of attrition,

3. Heightened stakeholder awareness of the nature of the material, with regard to particle attrition.

There has been a significant amount of research conducting into attrition of particulate materials in lean phase pneumatic conveying systems. A substantial amount of this research lies within the conveying of particulate food products. A number of models exist which seek to predict the level of erosion through modelling and empirical testing (1-4). This research is highly applicable to the current context as the failure of the pipe 
wall and the particulate material result from the same impact event. Modelling of particle breakage requires characterisation of the impact conditions, and the response of the material to these conditions.

It has been previously demonstrated that the attrition behaviour of a particle is unique to the material of which the particle is composed of.

The semi-brittle failure of cuboid crystals in the form of $\mathrm{NaCl}$ was investigated by Ghadiri and Zhang $(5,6)$ in considerable depth. Such particles were found to exhibit a chipping mechanism whereby platelets of material are removed from the corners of the particle.

Extensive work has been completed with regard to the compressive and impact strength of agglomerate materials in both experimental $(7,8)$ and simulation spheres (9-11). A considerable reliance on the agglomerate classification (wet, solid and binderless) which dictates the fracture behaviour of the overall structure, along with the distribution of internal bonds.

A number of approaches have been adopted to model this particle-wall impact process. Physical experiments have been carried out on gas blast $(12,13)$, centrifugal accelerator $(14,15)$, shear disc $(16)$, single bend pipeline $(16,17)$ and full scale pneumatic conveying equipment $(18,19)$. Each approach as attempted to identify either process or material characteristics affecting the attrition behaviour observed within full-scale systems. A number of approaches have also utilised a simulationbased approach making use of the Discrete Element Method (DEM) $(9,20)$, Finite Element Method (FEM) $(21,22)$, or through coupling with Computational Fluid Dynamics (CFD) (23). A stochastic approach to the characterisation of particle impacts at the pipe bend of a pneumatic conveying line was produced by Hanley et al. (24) 
which were compared to Monte Carlo simulations with good results for an idealistic system.

Key findings with respect to the material properties from these works are summarised as follows:

- Material properties of the particulate material have thus-far not been able to be linked with attrition behaviour through holistic relationships. Properties such as Young's Modulus, hardness, fracture toughness and tensile strength, coupled with micromechanical modelling techniques have not proven sufficient to quantitatively predict particle attrition behaviour (25).

- Strain rate is exceptionally important when testing particulate materials for attrition behaviour. Measurements such as material hardness by nanoindentation and yield strength have been proven to be strain-rate dependant and irreconcilable with the equivalent quasi-static process (25-27).

- It is generally accepted that smaller particles have a reduced propensity for breakage in comparison to larger particles. An explanation for this is that larger particles contain more imperfections than smaller particles, which may propagate more readily under loading conditions $(28,29)$.

Key findings with respect to the process conditions from these works are summarised as follows:

- Some studies show that bends of a shorter radius over pipe diameter ratio have been found to cause higher attrition levels than long-radius bends (30). Others agree with this trend however noting minimal difference between the two geometries (17). 
- Higher conveying air velocities (and hence higher particle velocities) caused increased attrition levels $(14,15,30,31)$.

- The higher the number of repeated loads, the greater the attrition observed (particle fatigue) $(18,19,32)$

- A marginal decrease in particle attrition has been found when particles are conveyed through two 45 degree bends as opposed to a single 90 degree bend (33).

\section{Method}

Section 3 will describe the methods used to acquire the results in this course of research.

\subsection{Materials}

Two materials were selected: golden breadcrumbs, and cooking salt. As illustrated in Figure 1 and Figure 2, the salt has a cubic shape, whereas the Golden Breadcrumbs have an angular shape. The former has a non-crystalline structure and the latter exhibits a crystalline structure. In addition, each material has a significantly different size distribution, with respect to the mean and range of particle sizes.

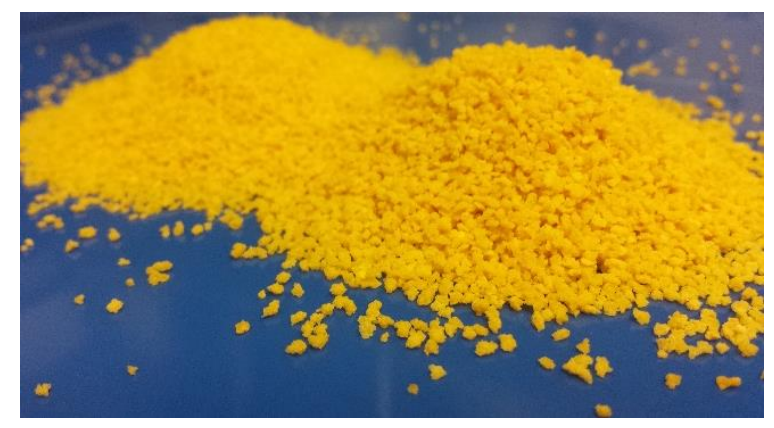

Figure 1 Golden Breadcrumb Particles 


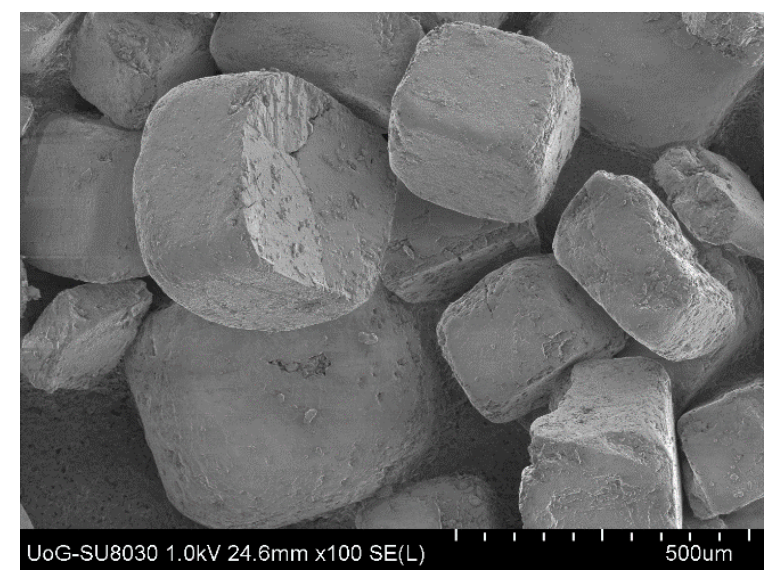

Figure 2 Scanning Electron Microscope Image of Salt Particles A 2D particle shape analysis was conducted on the materials, the results of which are summarised in Table 1. The values given represent the arithmetic mean of all particles analysed from a statistically representative sample of the master batch.

Table 1 Particle Shape Analysis. Standard Deviations of Values are given in Parentheses.

\begin{tabular}{|l|l|l|l|}
\hline \multicolumn{1}{|c|}{ Material } & \multicolumn{1}{|c|}{ Aspect Ratio } & $\begin{array}{c}\text { Equivalent Circle } \\
\text { Diameter }(\mu \mathrm{m})\end{array}$ & \multicolumn{1}{c|}{ Circularity } \\
\hline $\begin{array}{l}\text { Golden } \\
\text { Breadcrumbs }\end{array}$ & $0.685(0.166)$ & $95.16(133.14)$ & $0.718(0.175)$ \\
\hline Salt & $0.710(0.147)$ & $18.62(35.65)$ & $0.852(0.111)$ \\
\hline
\end{tabular}

\subsection{Statistically Representative Sampling}

Due to the generally highly variable nature of bulk materials, it was essential that the testing be conducted using statistically representative samples obtained from a master batch. Should this prerequisite be neglected, subsequent tests could be conducted using samples with significant variations in size distribution or mixture composition (34). Therefore, statistical subdivision was conducted through the use of two apparatuses: a spinning riffler and riffle boxes. 


\subsubsection{Spinning Riffler}

This piece of equipment was used to statistically subdivide samples of up to 40 litres in volume and is depicted in Figure 3.

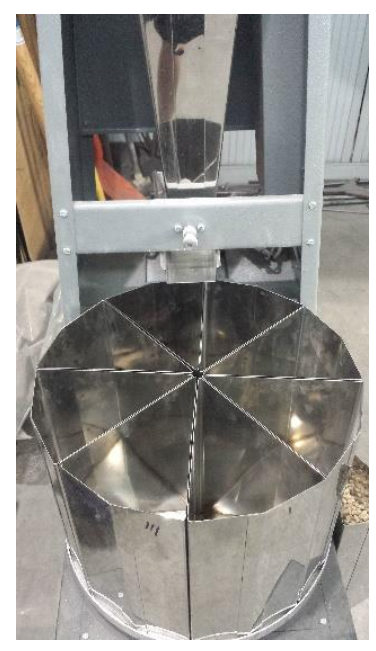

Figure 3 Spinning riffler

A mass-flow hopper positioned above a vibratory feeder that dispensed the master bulk sample into a rotating carousel, to create eight sub-samples. The amplitude of the vibratory feeder was varied to control the bulk material dispensary rate. The method produced eight statistically representative samples from a master sample.

\subsubsection{Riffle Boxes}

Riffle boxes were used for statistical subdivision of sample sizes up to approximately 500 millilitres. Figure 4 depicts the riffle boxes used for this programme of research.

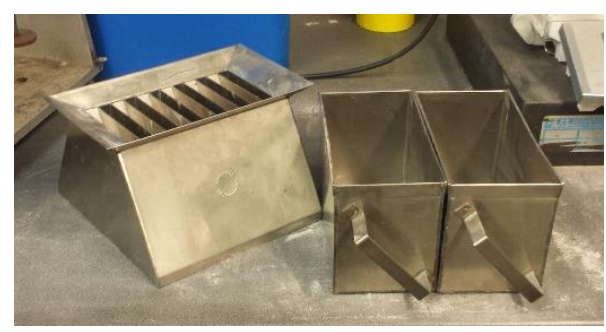

Figure 4 Riffle Boxes 
Material was poured into the top of the separating section, which contained a series of slots that alternated between two receiving vessels underneath. This method produced two statistically representative samples from a master sample.

\subsection{Particle Size Measurement}

In order to determine the virgin size distribution of the sample and the post-test size distribution, a stack of mechanical sieves was used. These were cleaned in an ultrasonic bath prior to use in order to remove any contaminants.

Due to the interest in the smaller size fractions generated, as detailed in the introduction, the sieve progression will begin at the smallest size of $45 \mu \mathrm{m}$. This will enable closer inspection of the material residing within small size fractions generated as a result of the degradation testing.

The bulk material sample was loaded into the top of the sieve stack, after which the stack was placed on a vibrating plate. The subsequent sieving was performed for 15 minutes.

Once sieving was complete, a set of electronic scales were used to determine the mass retained on each sieve to the nearest $0.01 \mathrm{~g}$.

\subsection{Attrition Test}

The attrition testing was performed on a centrifugal accelerator style attrition tester, depicted in Figure 5. 


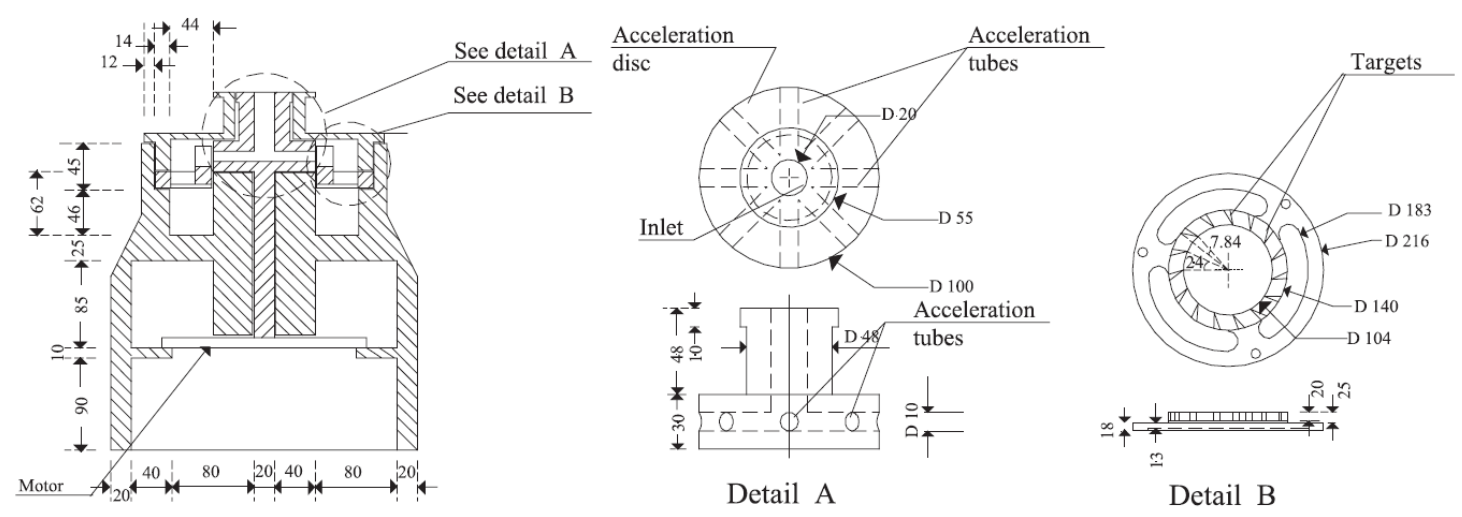

Figure 5 Schematic of Centrifugal Accelerator Attrition Tester (14)

The sample material was poured into the upper mass-flow hopper of the apparatus. It was then fed into the tester below via an increasing-capacity screw. The rate of screw rotation was kept constant throughout the course of the testing, and consistency in material delivery rate was stable. The function of the screw feeder was to ensure that the volumetric flow rate of material into the attrition tester was controlled - a trait that is not closely controlled in many forms of attrition testing $(15,35)$ as presented in Section 2.

The material fed from the screw feeder then entered the centre of a rotating spindle, and was accelerated outwards through a series of radial tubes. The accelerated particles then impact with a target array at a known angle of impact.

The post-test samples were collected at the base of the impact chamber, and taken for size analysis.

\subsubsection{Sample Preparation}

To obtain the required sample size for each test, the master sample was riffled down to a suitable size for separation via mechanical sieving. In the knowledge that each material batch being fed into the sieving unit was statistically representative of the master sample, the size fraction retained on each sieve may also be treated as 
statistically representative sample. Therefore, samples of known size range with upper and lower bounds may be prepared for testing in the centrifugal accelerator attrition tester.

\section{Results}

The results for this course of research consist of the initial virgin size distribution, the resultant size distributions when a statistically representative sample was degraded under known conditions, and the resultant size distribution when a known size fraction of material was degraded under known conditions.

It should be noted that the value of ' $1 \mu \mathrm{m}$ ' for the sieve size represents the material retained in the pan at the base of the sieve stack.

\subsection{Virgin Size Distribution}

The initial analysis undertaken was to determine the virgin size distribution of the 16 kg master sample for golden breadcrumbs and salt.

No particles used were retained on a $2000 \mu \mathrm{m}$ sieve - the next sieve in the progression.

Though inspection of the size distribution of golden breadcrumbs, the three size fractions representing the largest proportions of material by mass were chosen to undergo narrow size fraction degradation tests. These size fractions consisted of the material retained on 500, 710 and $1000 \mu \mathrm{m}$ sieves. These fractions represented approximately $58 \%$ of the total sample distribution by mass.

Through an inspection of the size distribution of salt, two size fractions, 250 and 355 $\mu \mathrm{m}$, were chosen for narrow size fraction degradation testing. These fractions represented approximately $75 \%$ of the total sample distribution by mass. 


\subsection{Size Distribution Post-Breakage}

This section presents the results of the degradation tests performed with the full virgin size distribution as the input material. Three velocities were tested: 15, 25 and $35 \mathrm{~m} / \mathrm{s}$. The results for the golden breadcrumbs are presented in Figure 6, and the results for the salt are presented in Figure 7 along with their respective virgin size distributions.

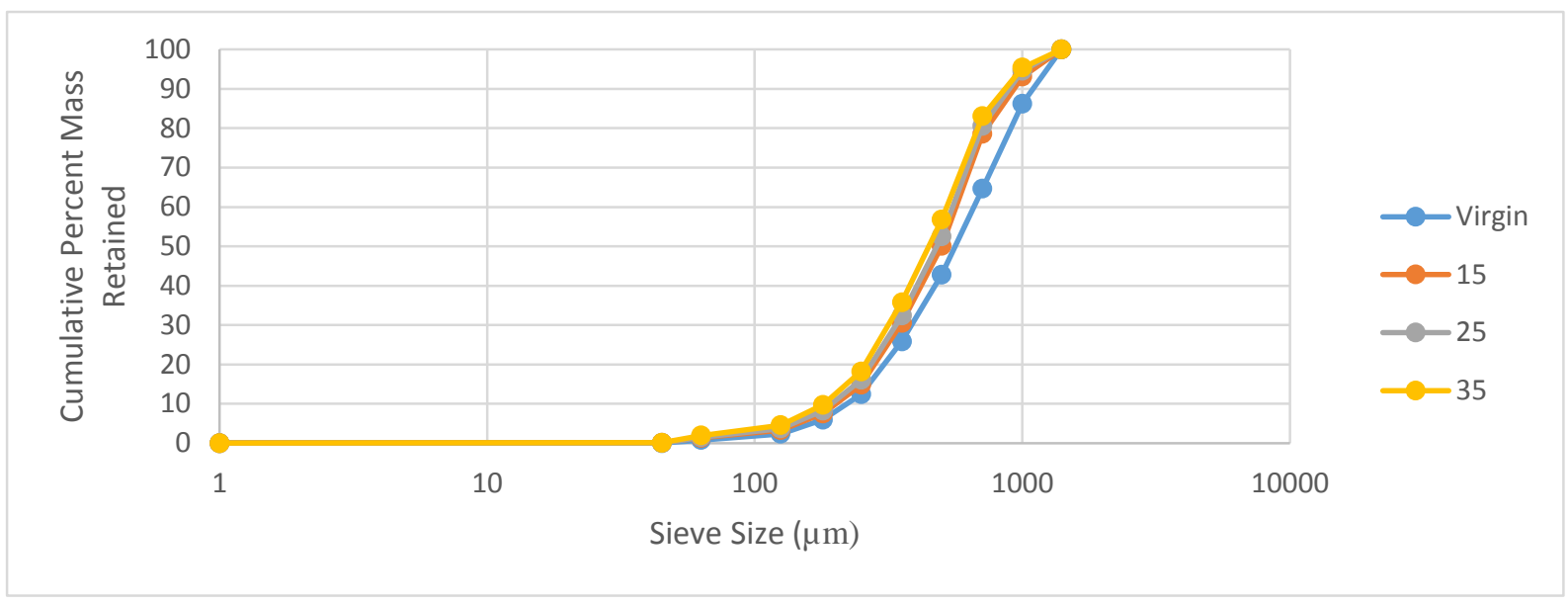

Figure 6 Full Size Distribution Breakage for Golden Breadcrumbs

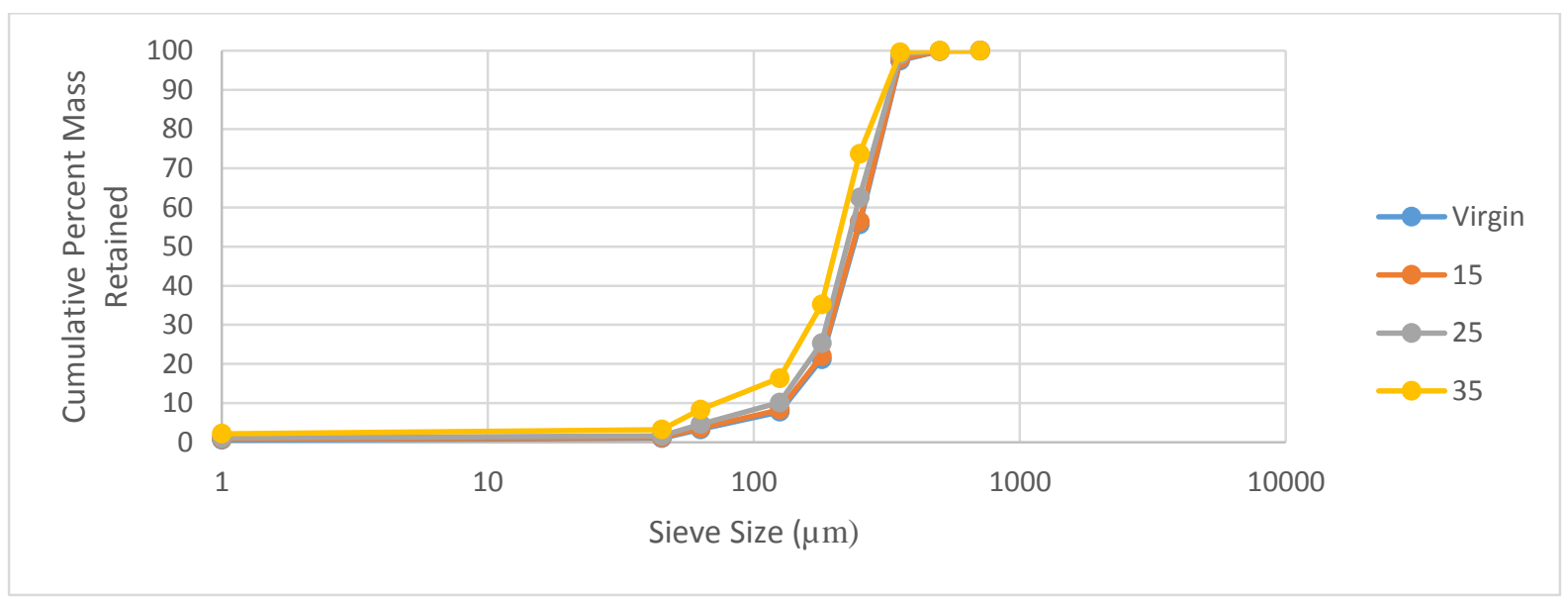

Figure 7 Full Size Distribution Breakage for Salt 


\subsection{Narrow-Fraction Size Distribution Post-Breakage}

This section presents the results of degradation tests of narrow size fractions across three impact velocities: 15,25 , and $35 \mathrm{~m} / \mathrm{s}$. Figure 8 presents the results for a 355 $500 \mu \mathrm{m}$ salt sample, all at impact velocities of 15,25 and $35 \mathrm{~m} / \mathrm{s}$.

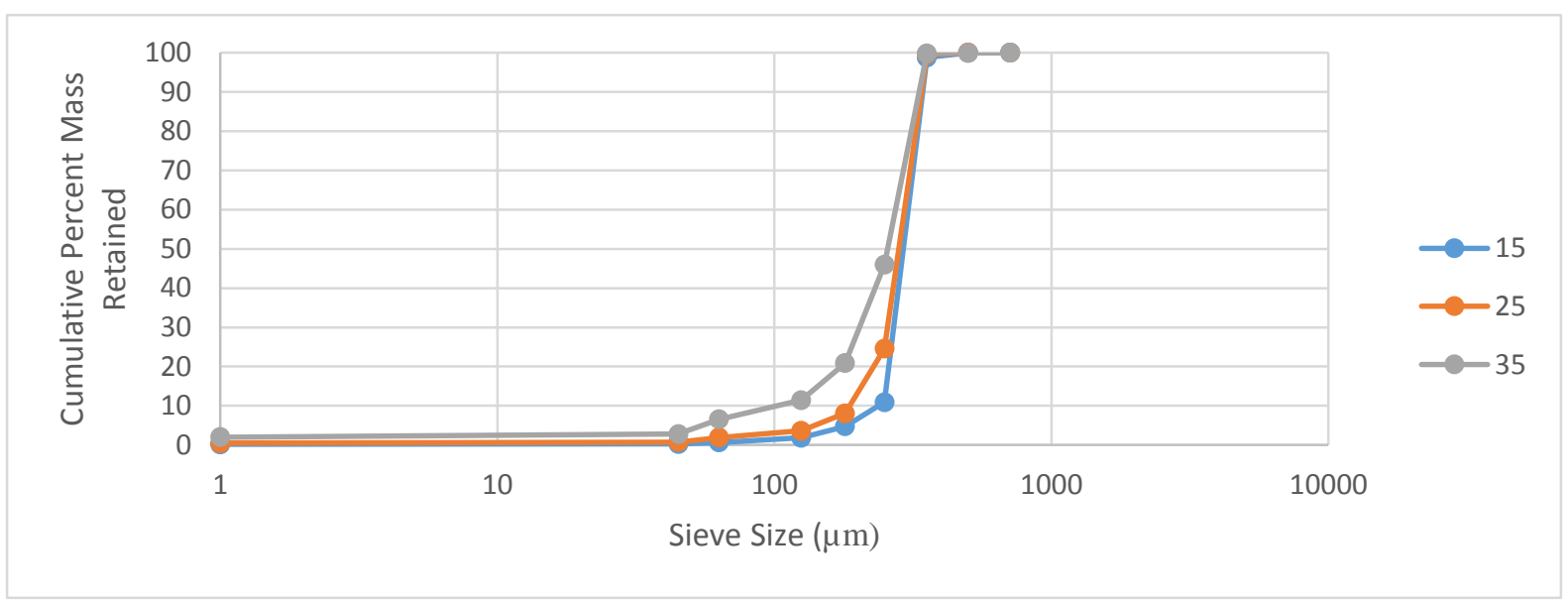

Figure 8 355-500 $\mu \mathrm{m}$ Narrow Size Fraction Breakage for Salt

This test used an input sample of $100 \%$ by mass $355-500 \mu \mathrm{m}$ material. Similar tests were also conducted with the size fractions specified in Section 4.1.

\section{Discussion}

This section will present the analysis of the results, looking at each of the materials and their representative size fractions in turn.

\subsection{Virgin Size Distribution}

There are considerable differences observable between the virgin size distributions of the subject materials in this research. The first of these differences is that the golden 
breadcrumbs exhibit a wider size distribution, where considerable sample mass is clearly spread across seven size fractions from 180 to $1400 \mu \mathrm{m}$. Additionally, smaller size fractions are represented with smaller mass percentages. In comparison, the salt exhibited a much narrower overall size distribution, with $75 \%$ of the sample by mass residing in two size fractions - on the 250 and $355 \mu \mathrm{m}$ sieves. Table 2 shows the $D_{10}$, D50, and D90 sizes for each material. Values were linearly interpolated from collected data to the nearest micron. The mass retained on a given sieve was assumed to have a mean size equal to the mean of the sieve size on which the material is retained and the smallest sieve size through which the material passed.

Table $2 D_{10}, D_{50}$, and $D_{90}$ Particle Sizes for each Material Tested

\begin{tabular}{|l|c|c|c|}
\hline \multicolumn{1}{|c|}{ Material } & D $_{10}$ & D50 & D90 \\
\hline Golden & 270 & 687 & 1337 \\
Breadcrumbs & & & 405 \\
\hline Salt & 163 & 288 & \\
\hline
\end{tabular}

\subsection{Full Size Distribution Tests}

Observations of the full size distribution tests displayed behaviour typical of attrition tests for most common materials. In the larger size fractions, more mass was retained for low impact velocities in comparison to the high impact velocities. This indicated that more of the larger particles in a sample broke under high impact energies. Additionally, it was evident that less mass was retained in the smaller size fractions at low impact velocities in comparison to the higher impact velocities. As expected, an increased number of smaller particles were generated at higher impact velocities. 
Figure 9 shows the percentage gain or loss in material for golden breadcrumbs in each size fraction, based on the virgin size distribution for each of the three impact velocities considered.

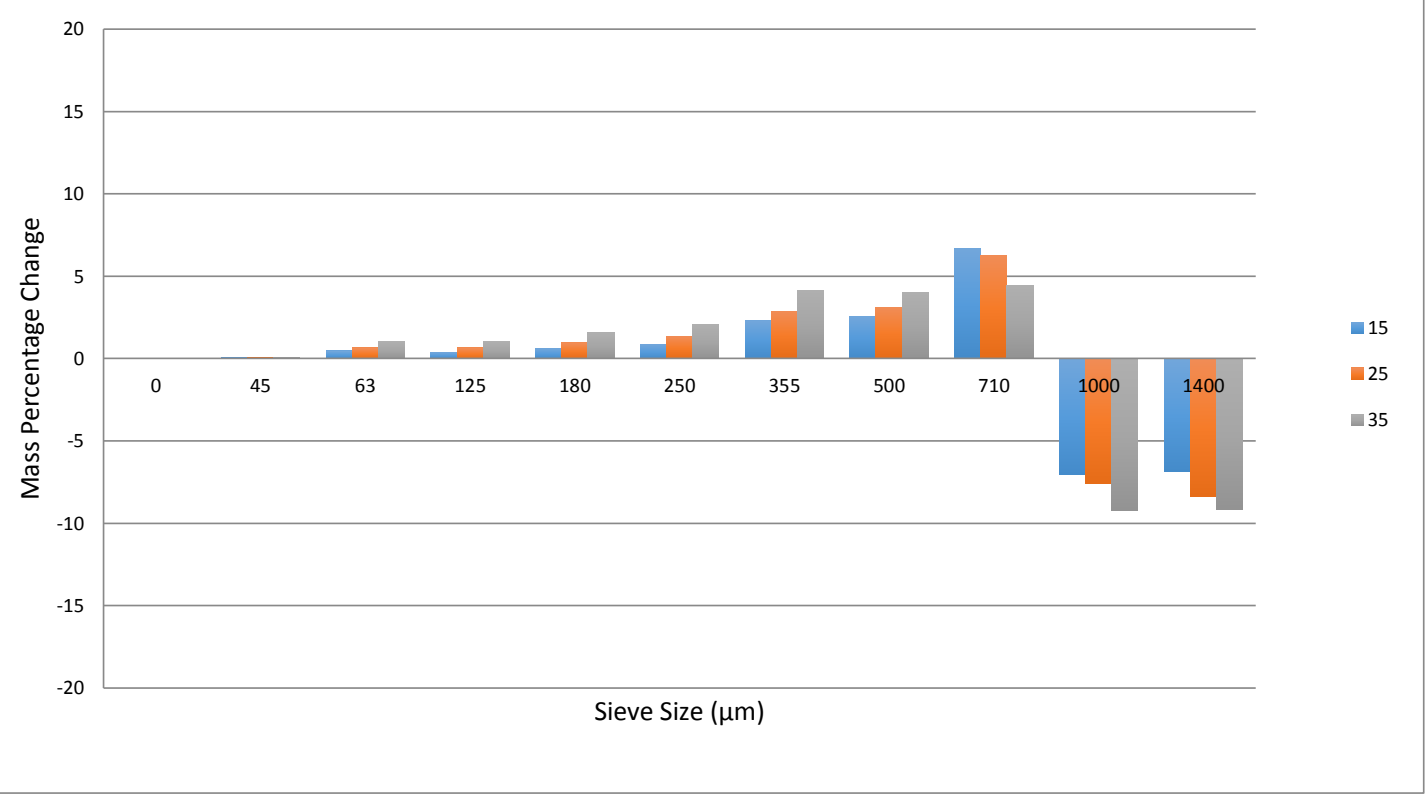

Figure 9 Percentage Mass Change for a Full Size Distribution Breakage of Golden Breadcrumbs

The only size fraction that does not follow the expected trend with regards to impact velocity, was the $710 \mu \mathrm{m}$ sieve. Rather than gaining more material as the impact velocity increased, the reverse was true. This may indicate that this particular size fraction loses more material through particle breakage, than it gains from particle breakage of larger size fractions, namely the 1000 and $1400 \mu \mathrm{m}$ fractions. By increasing the impact velocity, and hence the impact energy, this behaviour was emphasized. Figure 10 presents the results of the same analysis for salt. 


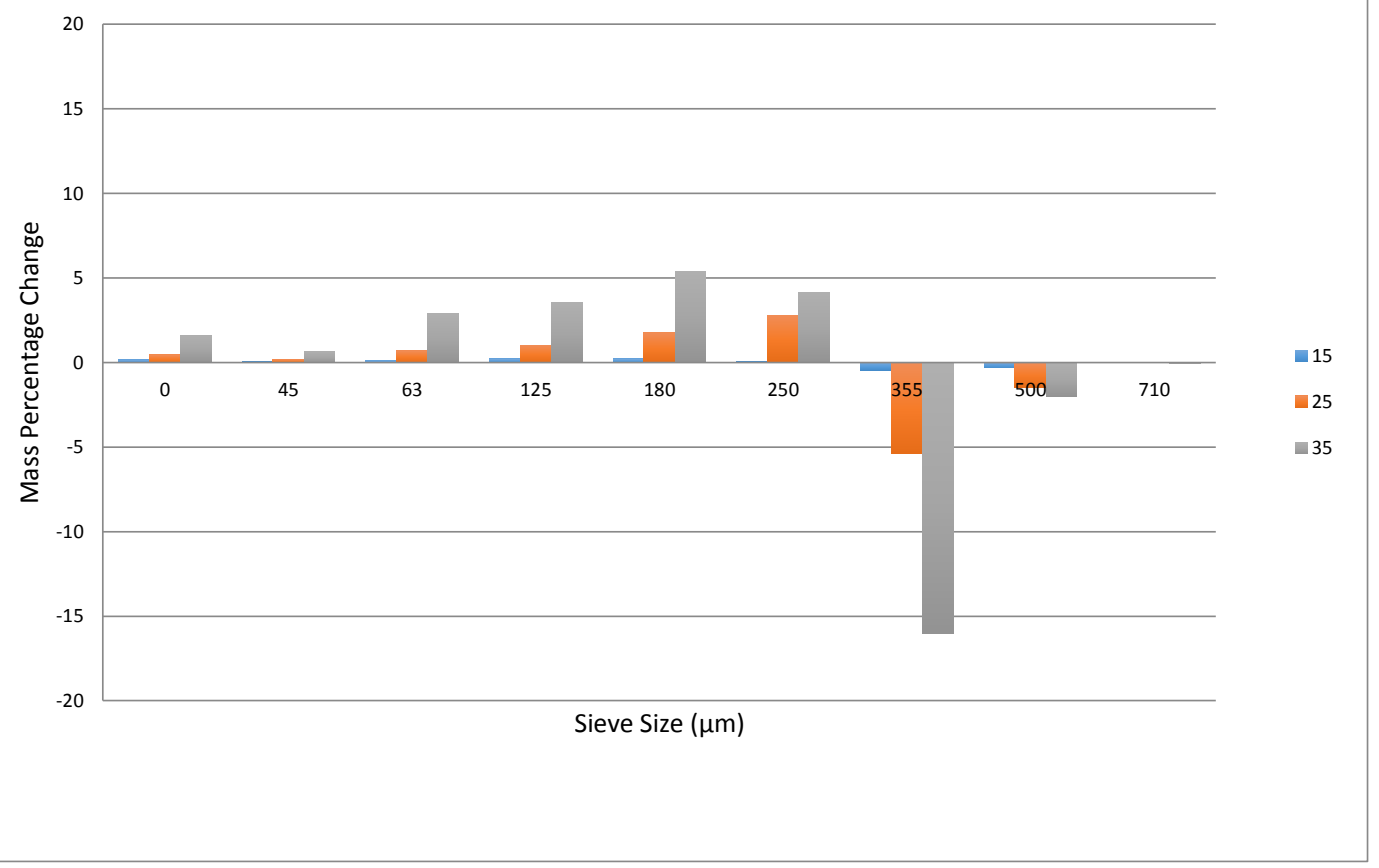

Figure 10 Percentage Mass Change for a Full Size Distribution Breakage of Salt

It was evident from the minimal migration of material across the size fractions at 15 $\mathrm{m} / \mathrm{s}$, that the impact energy was not sufficient to cause a significant amount of degradation. However, it can be concluded that at 25 and $35 \mathrm{~m} / \mathrm{s}$, the impact energy was sufficient to generate smaller particles from the larger size fractions. In particular, large changes were observed at $35 \mathrm{~m} / \mathrm{s}$, where a significant proportion of particles undergo size changes.

\subsection{Narrow Size Fraction Tests}

General observations of the narrow size fraction attrition tests clearly showed that larger particles were more susceptible to breakage than the smaller particles, for the materials studied. Additionally, the narrow size fractions displayed similar behaviour to that observed in the full size distribution tests, where more particles were broken at higher impact velocities. 
Figure 12, Figure 14 and Figure 16 show the percentage change in the size distribution for narrow size fractions of 1000-1400, 710-1000, and 500-710 $\mu \mathrm{m}$ for golden breadcrumbs respectively.

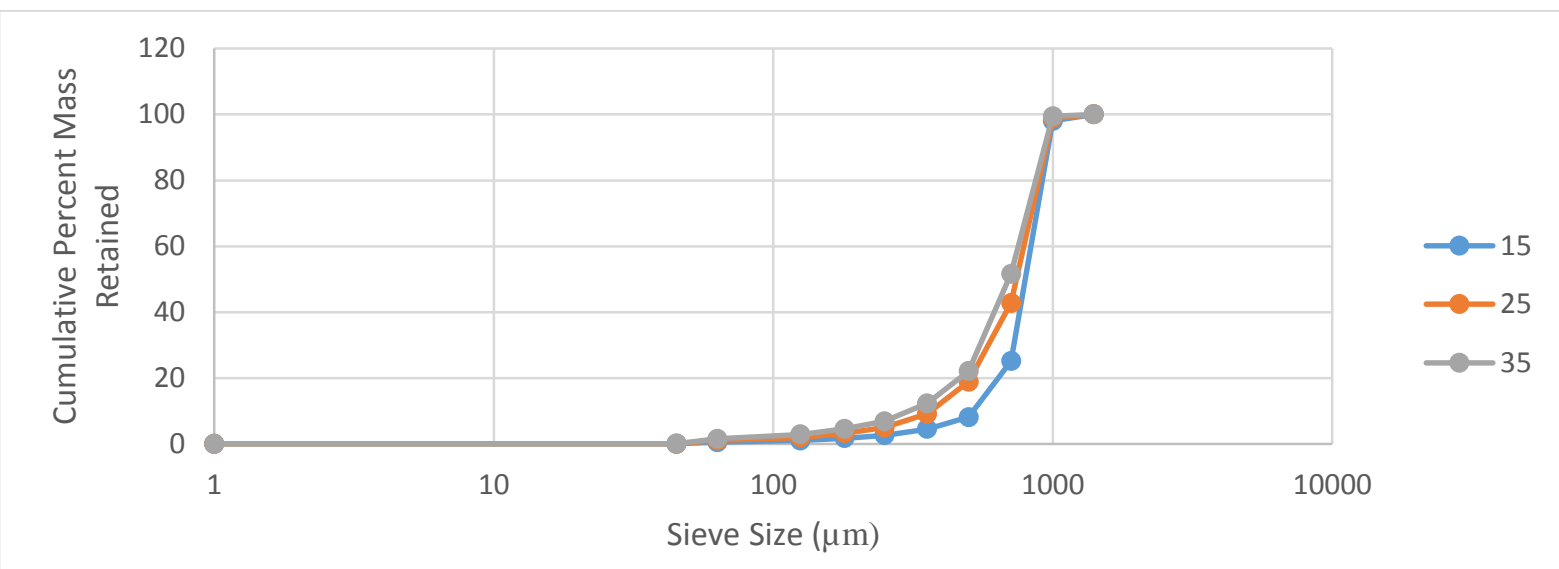

Figure 11 Cumulative Percent Mass Retained for a 1000-1400 $\mu \mathrm{m}$ sample of Golden Breadcrumbs across all Impact Velocities

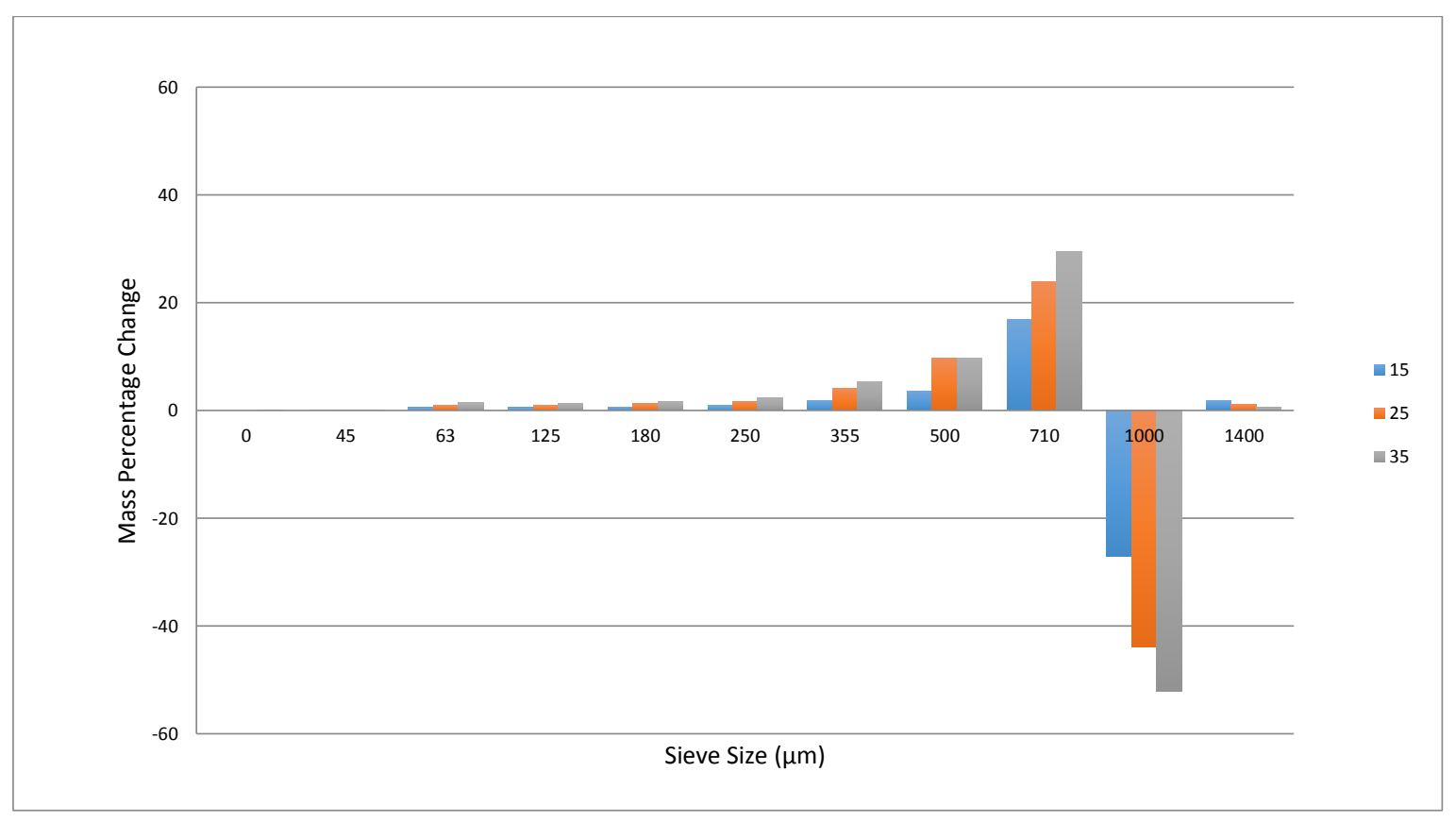

Figure 12 Percentage Mass Change for a 1000-1400 $\mu \mathrm{m}$ sample of Golden Breadcrumbs 


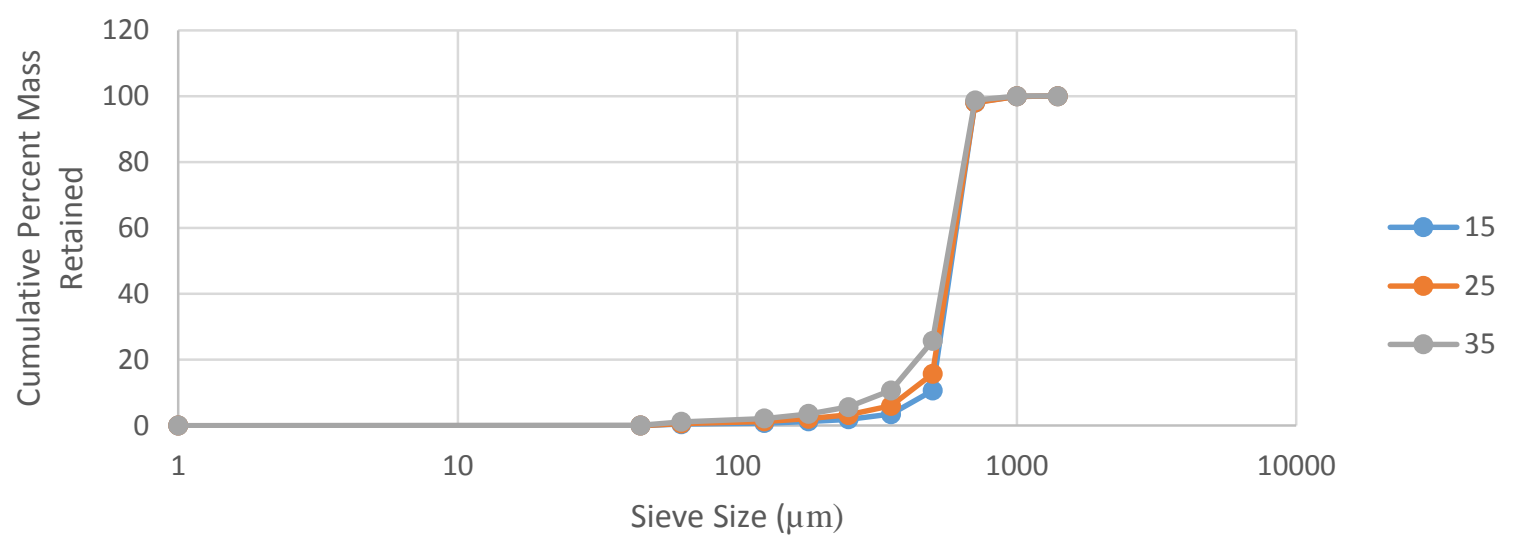

Figure 13 Cumulative Percent Mass Retained for a 710-1000 $\mu \mathrm{m}$ sample of Golden Breadcrumbs across all Impact Velocities

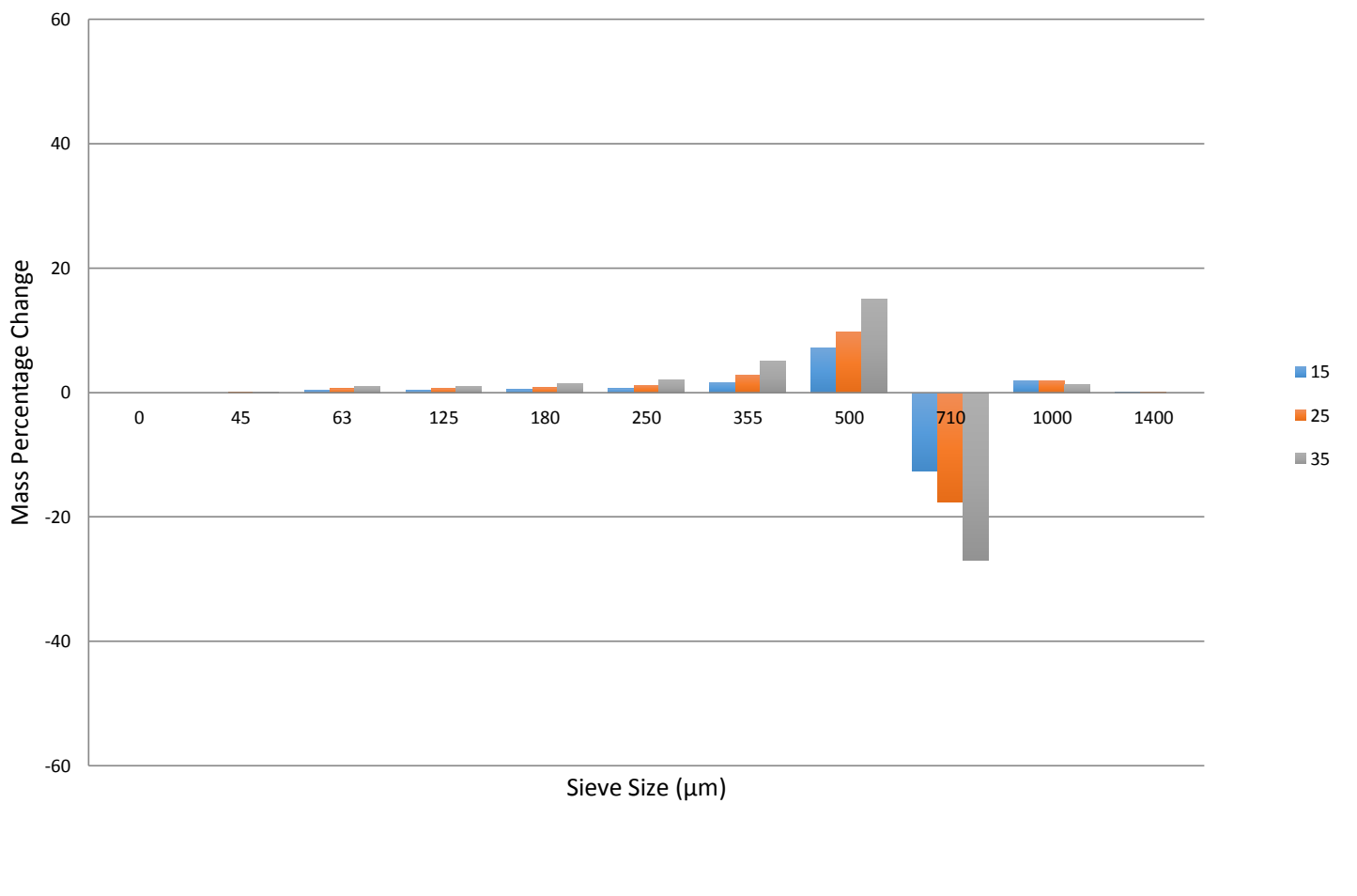

Figure 14 Percentage Mass Change for a $710-1000 \mu \mathrm{m}$ sample of Golden Breadcrumbs 


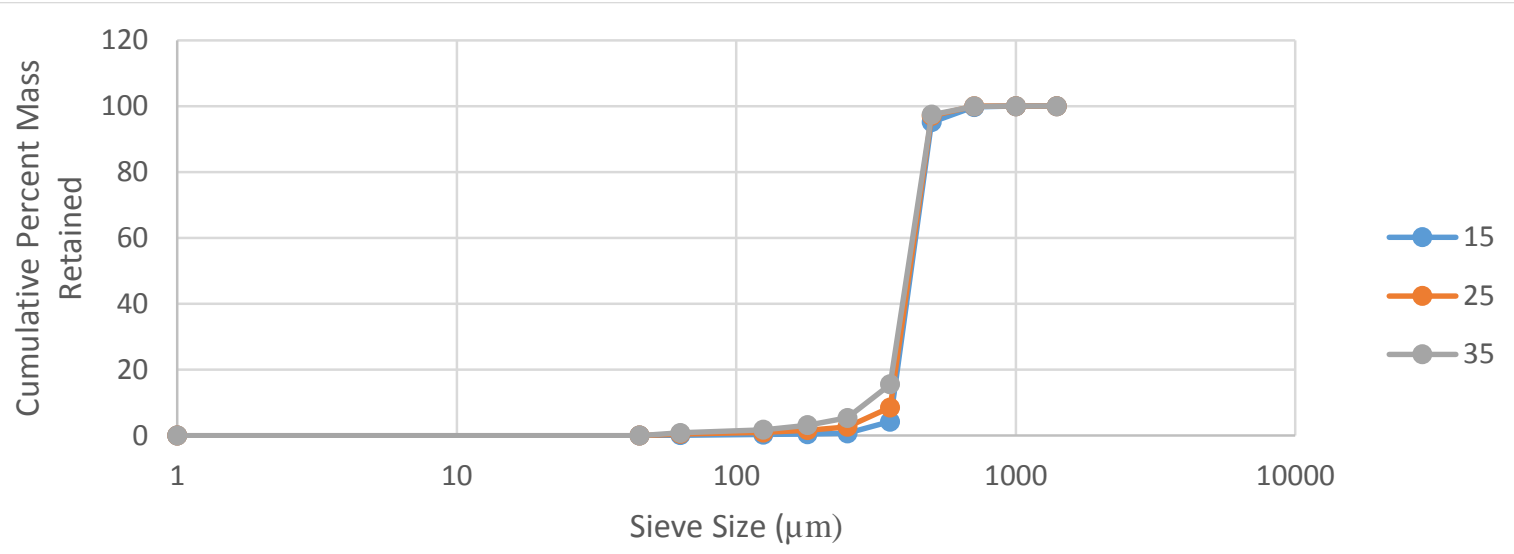

Figure 15 Cumulative Percent Mass Retained for a 500-710 $\mu \mathrm{m}$ sample of Golden Breadcrumbs across all Impact Velocities

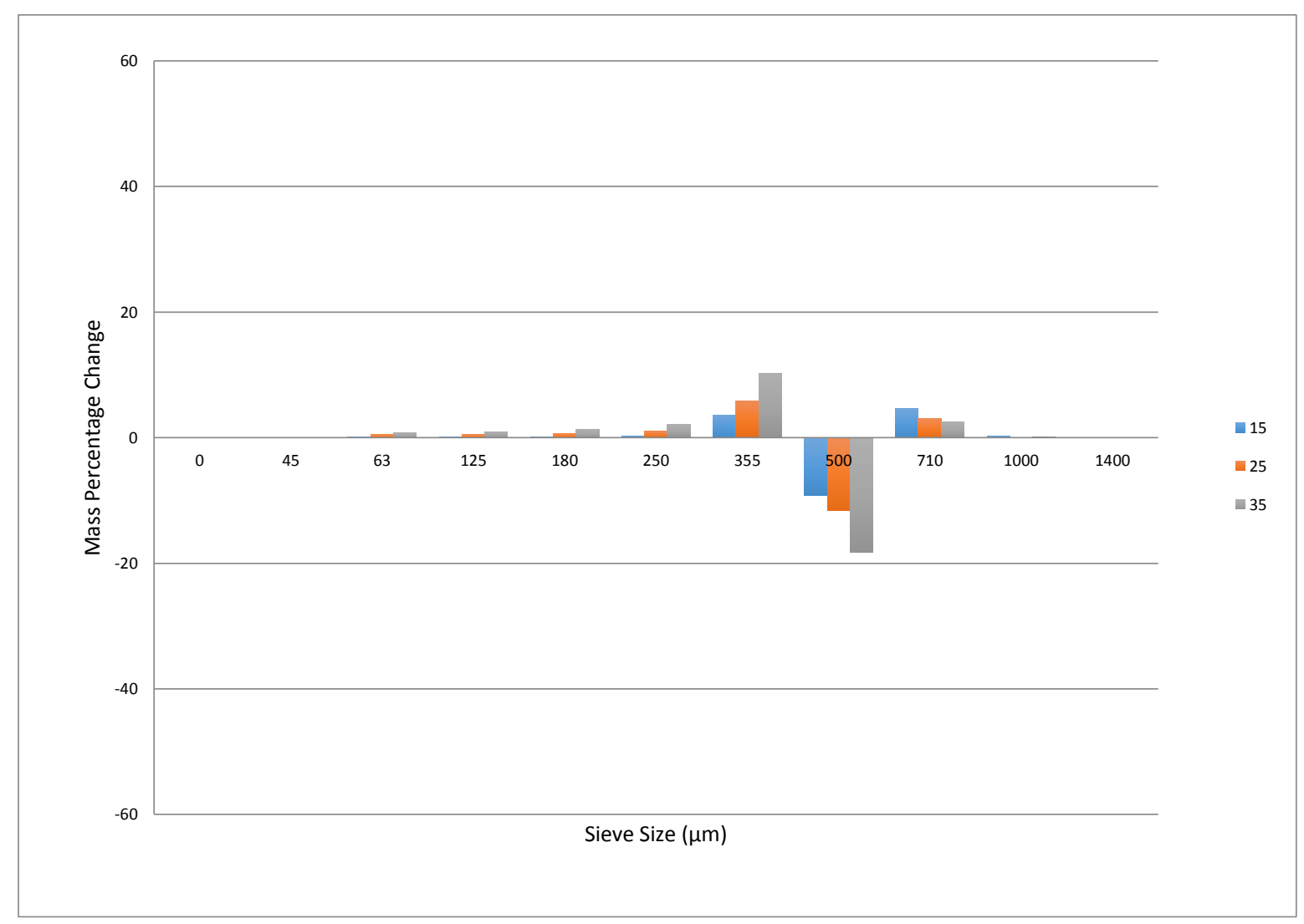

Figure 16 Percentage Mass Change for a 500-710 $\mu \mathrm{m}$ sample of Golden Breadcrumbs

This analysis shows some mass retained on sieves larger than the size fraction under scrutiny. This was probably due to the irregular shape of the particles, which have diameters dependent on the orientation of the particle with respect to the sieve mesh. Another possible explanation was that the particles agglomerated in the testing and 
sieving phases of the experimentation. Further work is required to quantify this anomaly.

In comparing the analysis of three adjacent size fractions for golden breadcrumbs, particle sizes greater than $1000 \mu \mathrm{m}$ in diameter break to a considerably greater degree than either of those retained on 500 or $710 \mu \mathrm{m}$ sieves. This suggested that once particles reach $1000 \mu \mathrm{m}$ in diameter, the particle material does not have enough internal strength to withstand the momentum transfer of the impact. However, with the reduced diameter of the 710 and $500 \mu \mathrm{m}$ particles, the mass was sufficiently small to maintain the structure of more particles post-impact.

Figure 18 and Figure 20 show the mass percentage change analysis of the $355-500$ and 250-355 $\mu \mathrm{m}$ size fractions for salt respectively. They are preceded by their respective cumulative particle size distributions in Figure 17 and Figure 19.

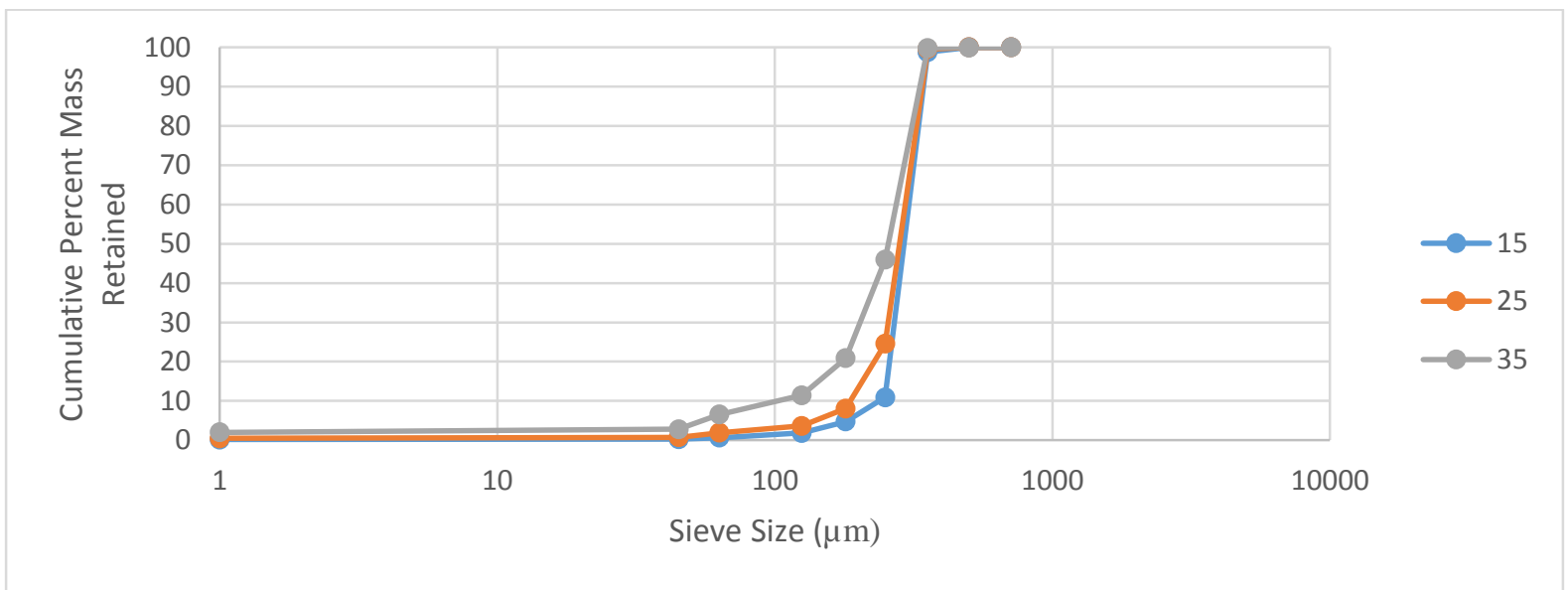

Figure 17 Cumulative Percent Mass Retained for a 355-500 $\mu \mathrm{m}$ sample of Salt across all Impact Velocities 


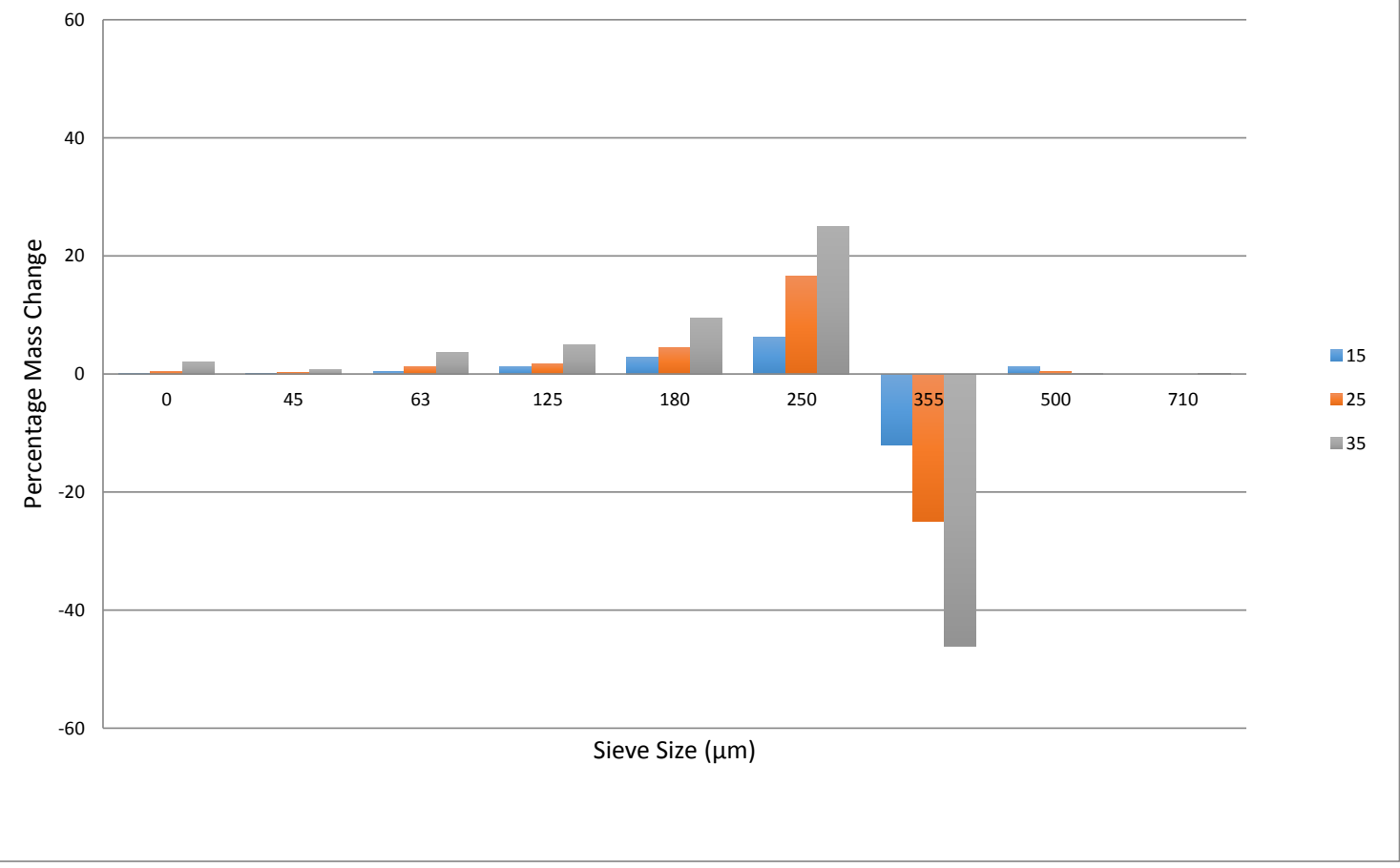

Figure 18 Percentage Mass Change for a $355-500 \mu \mathrm{m}$ sample of Salt

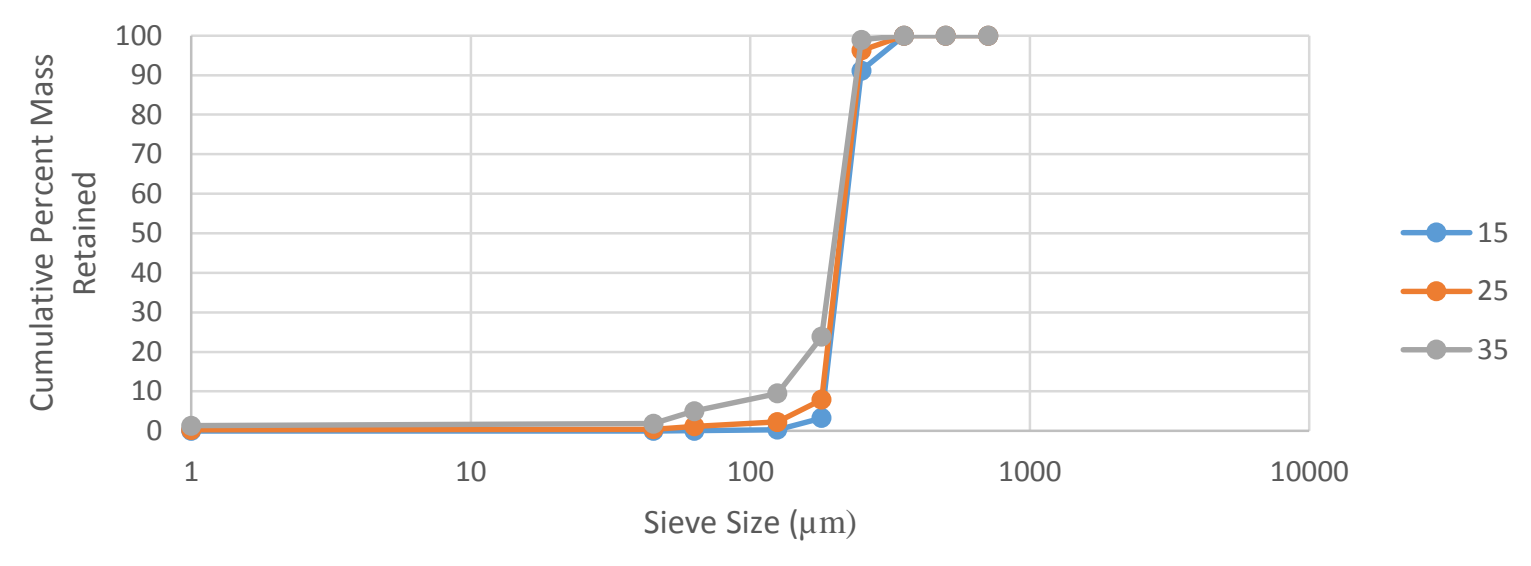

Figure 19 Cumulative Percent Mass Retained for a 250-355 $\mu \mathrm{m}$ sample of Salt across all Impact Velocities 


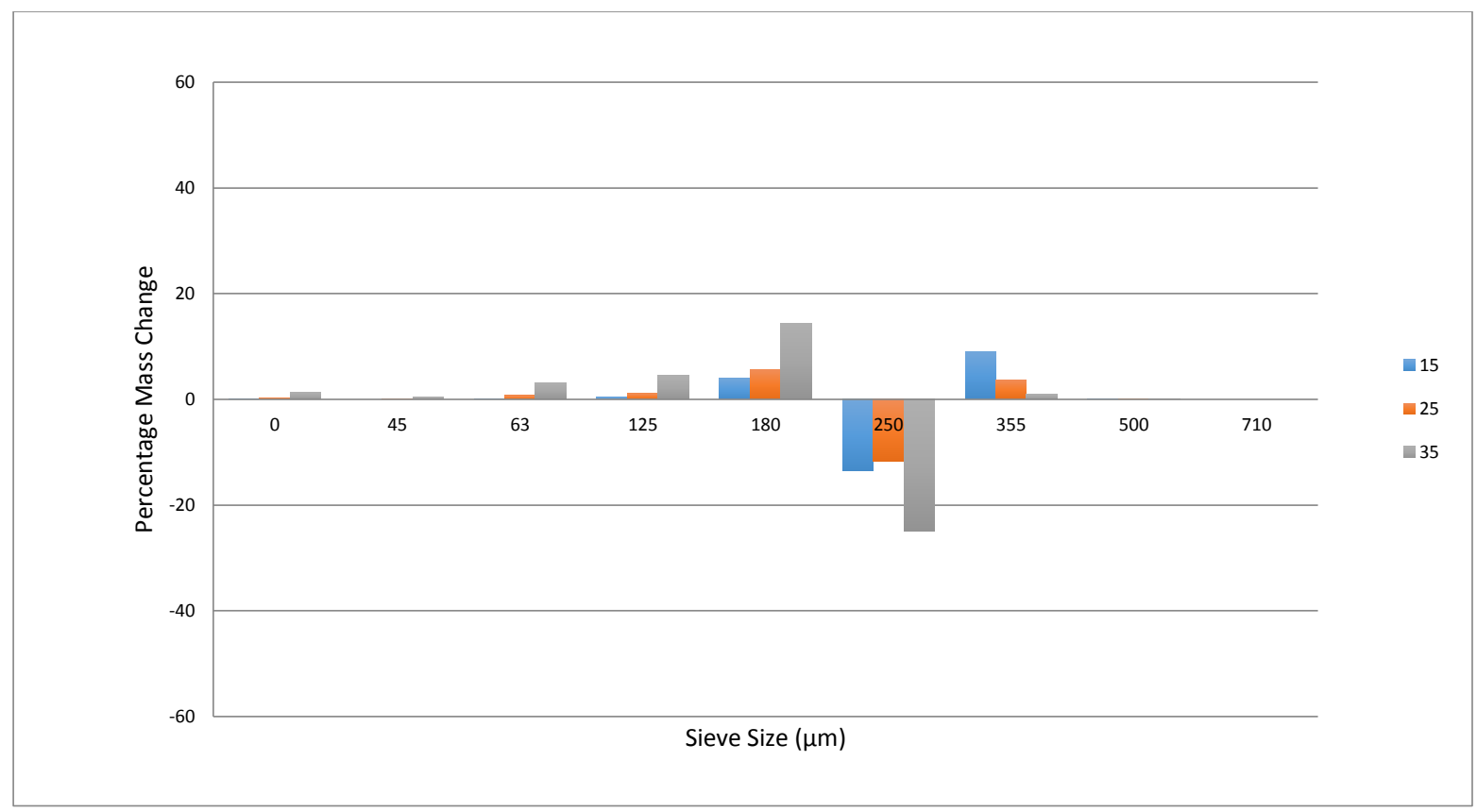

Figure 20 Percentage Mass Change for a 250-355 $\mu \mathrm{m}$ sample of Salt Even though the size fractions considered for salt were smaller than those considered for golden breadcrumbs, the magnitude of breakage occurring was comparable under some impact velocities. For example, the amount of material lost for salt in the $355 \mu \mathrm{m}$ size fraction tested at $35 \mathrm{~m} / \mathrm{s}$, was of the same order of magnitude as that lost for golden breadcrumbs in the $1000 \mu \mathrm{m}$ size fraction at $35 \mathrm{~m} / \mathrm{s}$. While there was little relationship between the two materials with respect to particle size, this demonstrates the influence of the material with regard to the particle strength.

\subsection{Particle Breakage Relationships}

This Section will address the observed relationships between the breakage behaviour observed across all tests.

\subsubsection{Breakage Matrix Analysis}

Breakage matrix analysis proceeds according to the method described by Chapelle (15). Equation (1) describes the mathematical approach underlying the method. 


$$
[B] \cdot[i]=[o]
$$

It is clear that the predictive capability of this approach heavily relies on the integrity of the breakage matrix, and subsequent selection of the correct breakage matrix pertaining to the conveying conditions.

The breakage matrix takes the form as depicted in Equation (2).

$$
[B]=\left[\begin{array}{ccccc}
b_{a a} & 0 & 0 & 0 & 0 \\
b_{b a} & b_{b b} & 0 & 0 & 0 \\
b_{c a} & b_{c b} & b_{c c} & 0 & 0 \\
b_{d a} & b_{d b} & b_{d c} & b_{d d} & 0 \\
b_{e a} & b_{e b} & b_{e c} & b_{e d} & b_{e e}
\end{array}\right]
$$

The subscripts on each element of the matrix denote the size fraction from which the material came and the size fraction that it arrives in post-breakage. For example, element $b_{d c}$ denotes the material going from size fraction $\mathrm{C}$, to size fraction $\mathrm{D}$. Note that the upper right hand corner of the matrix are zeros, as particles are not expected to increase in diameter.

The analysis performed in this research will use the empirical data collected to generate the breakage matrix for each of the conditions tested.

There are numerous advantages in testing the individual size fractions of a bulk material sample. One such advantage is that if there is a change in the composition of size fractions of the stock product, the breakage relationships may be recalculated without having to re-test the new material. Additionally, if there is more than one bend in the pneumatic pipeline under investigation, the breakage matrix may be applied to 
the virgin size distribution the number of times corresponding to the number of bends. This compounded breakage will then yield an estimated output size distribution as a result of the entire pneumatic conveying line.

\subsubsection{Comparison of Simulated and Experimental Full Size Distribution Breakage}

This approach will make the following assumptions/limitations:

1. Material collected on sieves greater than the diameter of size fractions of interest in the narrow size fraction post-breakage measurements will be omitted and treated as an error in the experimental method,

2. Material in the virgin size distribution greater in diameter than that considered in narrow size fraction testing will be omitted from the results displayed, however will still be accounted for in overall sample size to calculate percentage masses of subsequent size fractions,

3. Material in size fractions smaller than those tested under the narrow size fraction tests will be treated as stationary (undergo no breakage in the simulation),

4. Only a limited number of size fractions will be considered in the testing regime, as addressed in Section 4.3, though it is acknowledged that for optimum results all size fractions should be tested.

5. Due to material losses throughout the course of experimentation, percentage mass retained for each sieve size of the Simulated Particle Size Distribution has been prorated. This accounts for the large size fractions omitted as part of the experimentation. 
Figure 21, Figure 22 and Figure 23 compare the experimental results for a full size distribution sample, post breakage (denoted by FSD) and the simulated results (denoted by SIM) for golden breadcrumbs at impact velocities of 15,25 , and $35 \mathrm{~m} / \mathrm{s}$ respectively.

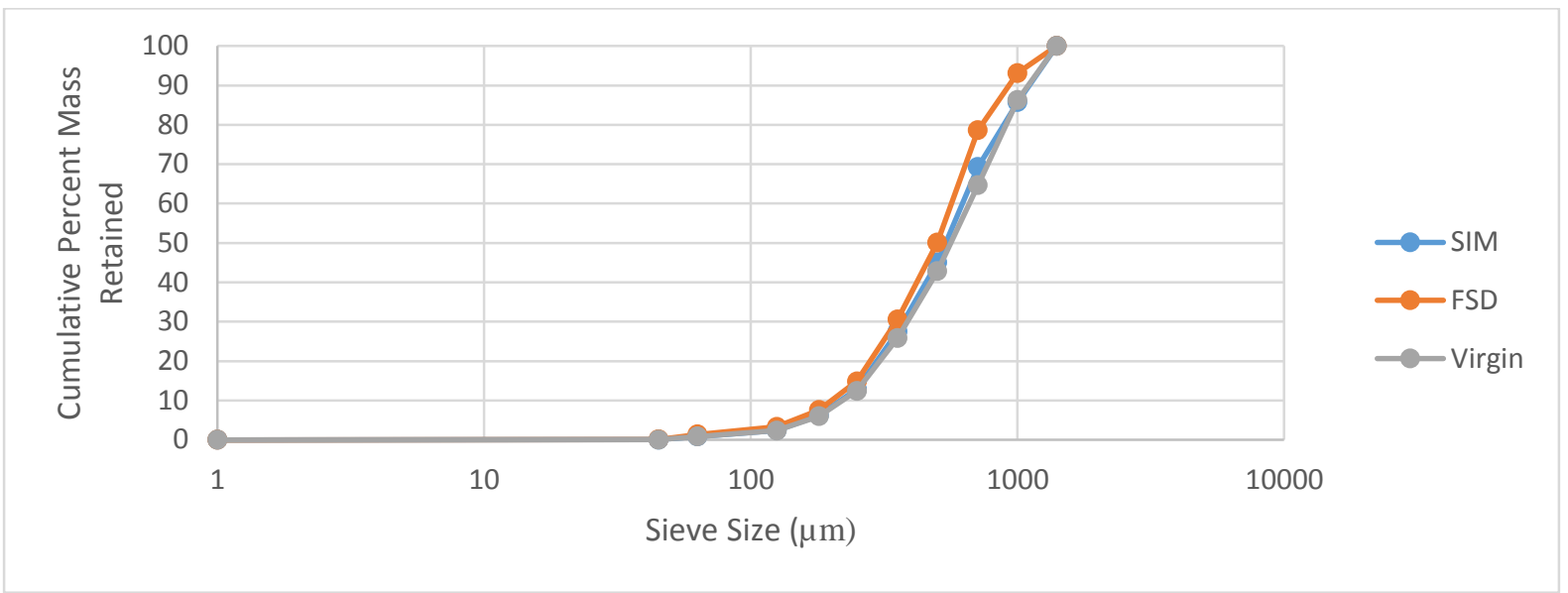

Figure 21 Simulated (SIM) and Full Size Distribution (FSD) Post-Breakage Results for Golden Breadcrumbs at $15 \mathrm{~m} / \mathrm{s}$ impact velocity

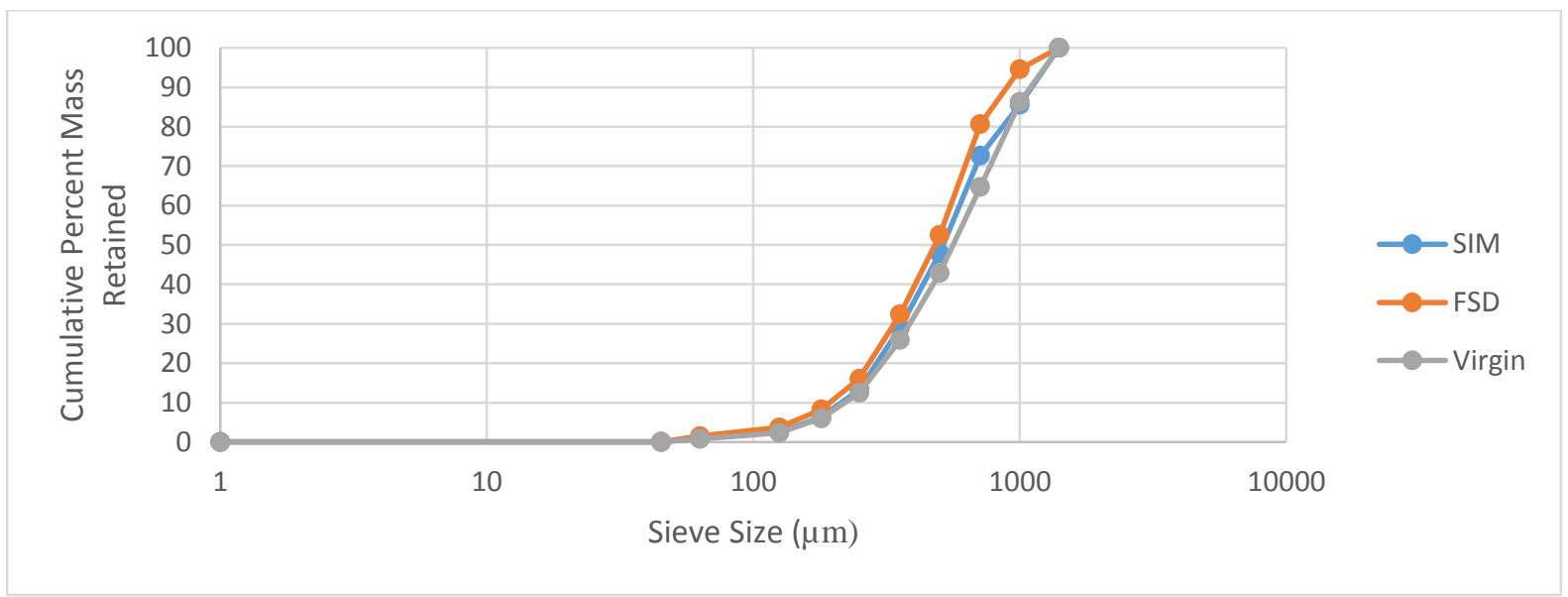

Figure 22 Simulated (SIM) and Full Size Distribution (FSD) Post-Breakage Results for Golden Breadcrumbs at $25 \mathrm{~m} / \mathrm{s}$ impact velocity 


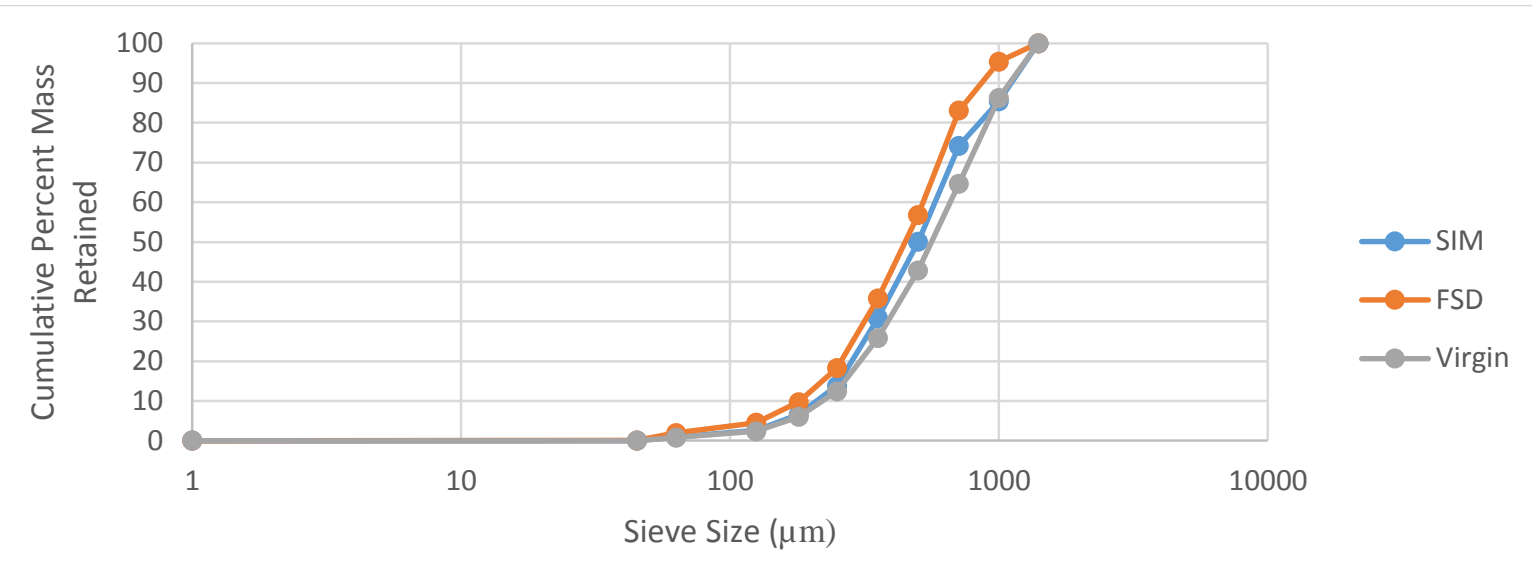

Figure 23 Simulated (SIM) and Full Size Distribution (FSD) Post-Breakage Results for Golden Breadcrumbs at $35 \mathrm{~m} / \mathrm{s}$ impact velocity

Considering the assumptions made in the simulation method, the predictions made fit reasonably well with the full size distribution breakage results. The error between the predicted attrition values and the experimental values was calculated as a percentage difference of the experimental cumulative mass retained. This error was not greater than $12 \%$ for all size fractions $355 \mu \mathrm{m}$ and greater. It is expected that significantly greater error values will be obtained for the finer particle size fractions due to the relatively small percentage of sample mass that they represent. The most notable differences that can be observed lie primarily in the larger size fractions, namely 710 and $1000 \mu \mathrm{m}$. This observation is expected, as the $1400 \mu \mathrm{m}$ size fraction was not considered in the narrow size fraction breakage analysis. It would be logically sound to state that this size fraction would contribute to the 710 and $1000 \mu \mathrm{m}$ fractions, increasing agreement between the simulated and experimental values. More detail is therefore required on the largest size fraction, with regard to both its size distribution and breakage behaviour. As a result of the findings here, it is recommended that future experiments consider narrow size fraction tests with the largest size fraction in all cases. 
With regard to the smaller size fractions, the simulation underestimates the magnitude consistently. This may be explained by the simulation failing to account for the movement of all size fractions below those for which results are presented. It is expected that all fractions will break to some degree, thus contributing to the stated error.

As a form of preliminary investigation, the analysis of the size fractions cumulatively containing in excess of $50 \%$ of the virgin size distribution by mass is sufficient in obtaining breakage data to a moderate accuracy.

By taking into consideration both of the aforementioned statements, the accuracy of the simulated predictions could be increased.

Figure 24, Figure 25 and Figure 26 compare the experimental results (denoted by FSD) and the simulated results (denoted by SIM) for salt, at impact velocities of 15 , 25 , and $35 \mathrm{~m} / \mathrm{s}$ respectively.

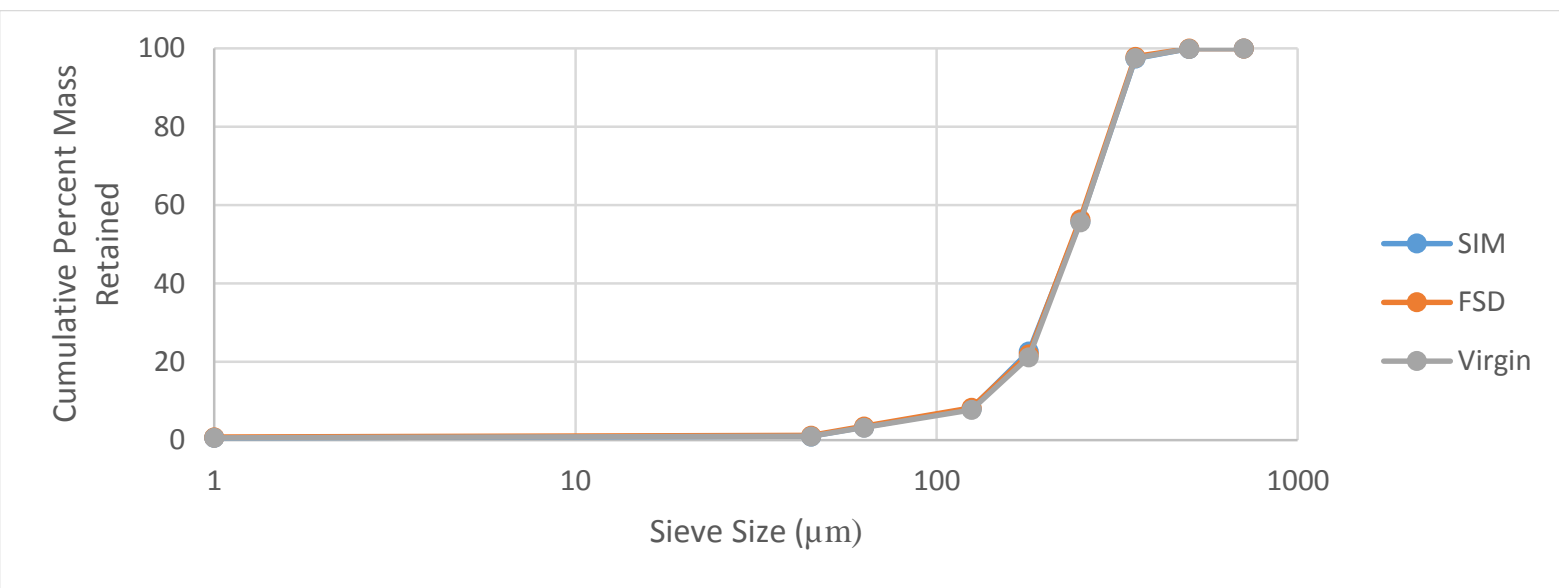

Figure 24 Simulated (SIM) and Full Size Distribution (FSD) Post-Breakage Results for Salt at $15 \mathrm{~m} / \mathrm{s}$ impact velocity 


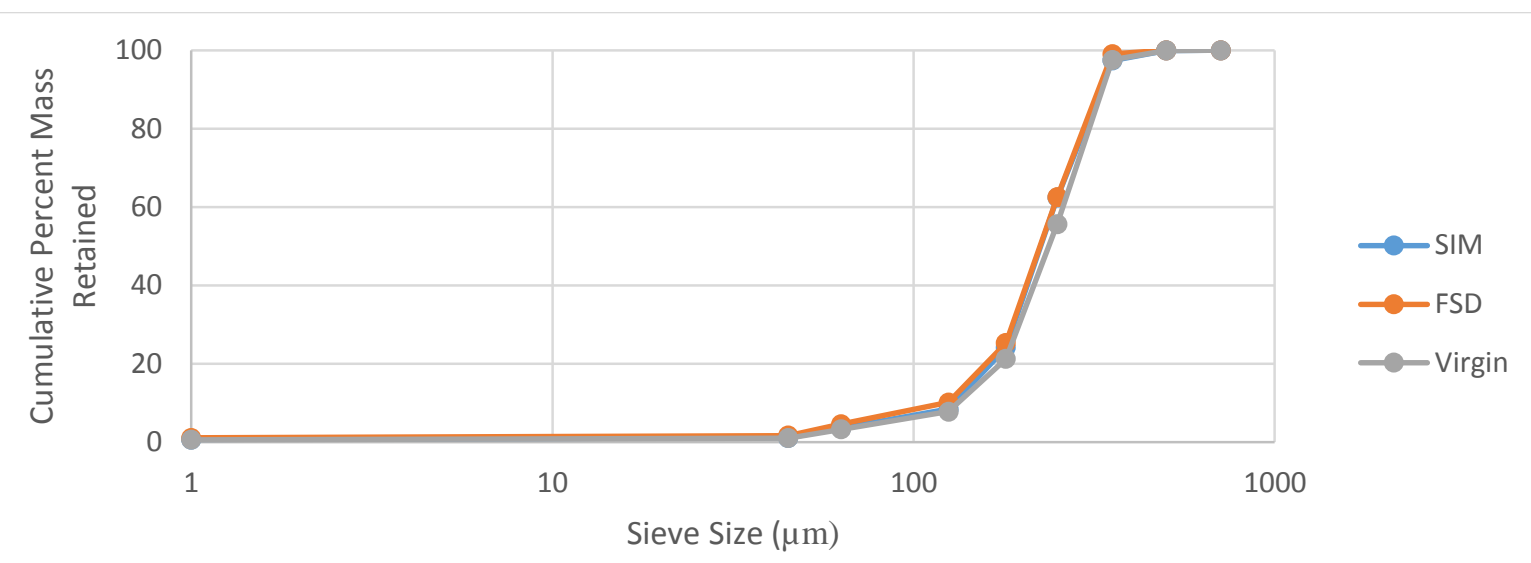

Figure 25 Simulated (SIM) and Full Size Distribution (FSD) Post-Breakage Results for Salt at $25 \mathrm{~m} / \mathrm{s}$ impact velocity

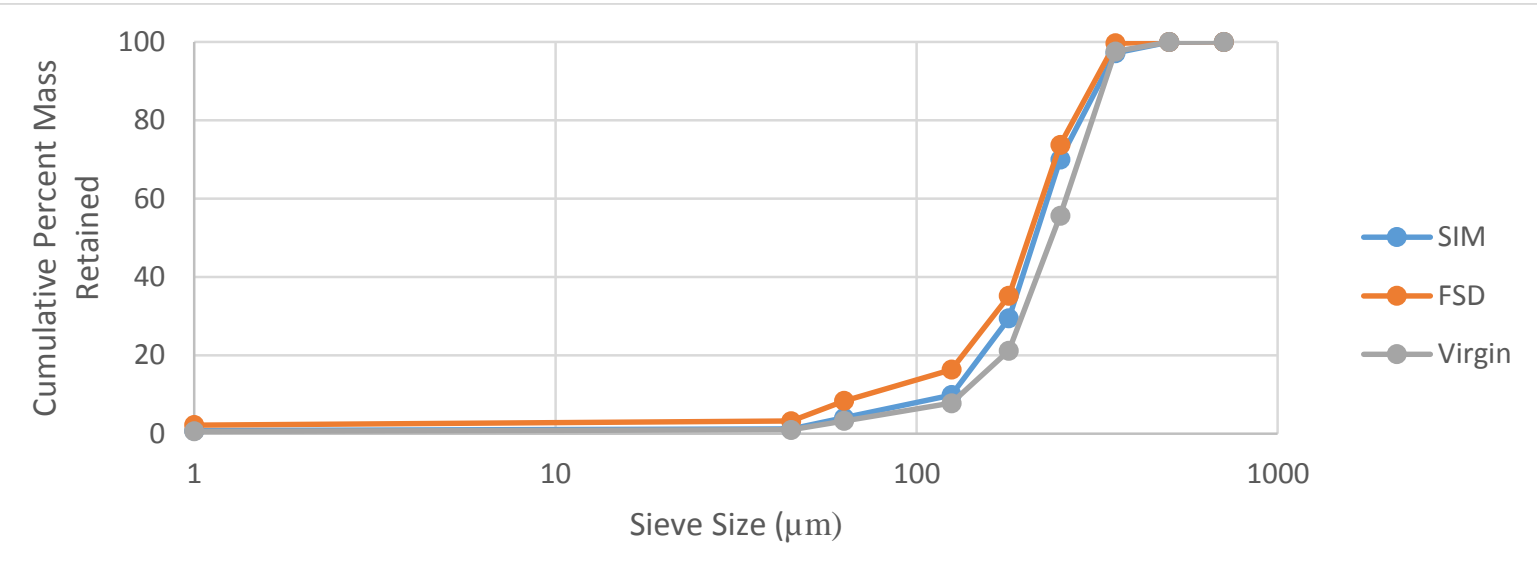

Figure 26 Simulated (SIM) and Full Size Distribution (FSD) Post-Breakage Results for Salt at $35 \mathrm{~m} / \mathrm{s}$ impact velocity

As with the golden breadcrumbs, a good agreement between the simulated and experimental results for salt is achieved. Almost all size fractions are under-predicted once again, and as with the golden breadcrumbs, the logical explanations for this behaviour lies with; the omission of the size fractions larger than those tested, and to the migration of smaller size fractions. The error was calculated in the same manner as with the Golden Breadcrumbs and it was found that all size fractions $250 \mu \mathrm{m}$ and greater had equal to, or less than $5 \%$ error. 


\subsection{Particle Size, Impact Velocity and Breakage Behaviour Relationships}

Researchers have reported in the literature that as particle size reduces, particle strength increases $(36,37)$. Figure 27 shows the percentage mass decrease with regard to impact velocity, particle size and material type. The dashed line denotes Salt and the solid line denotes Golden Breadcrumbs (GB).

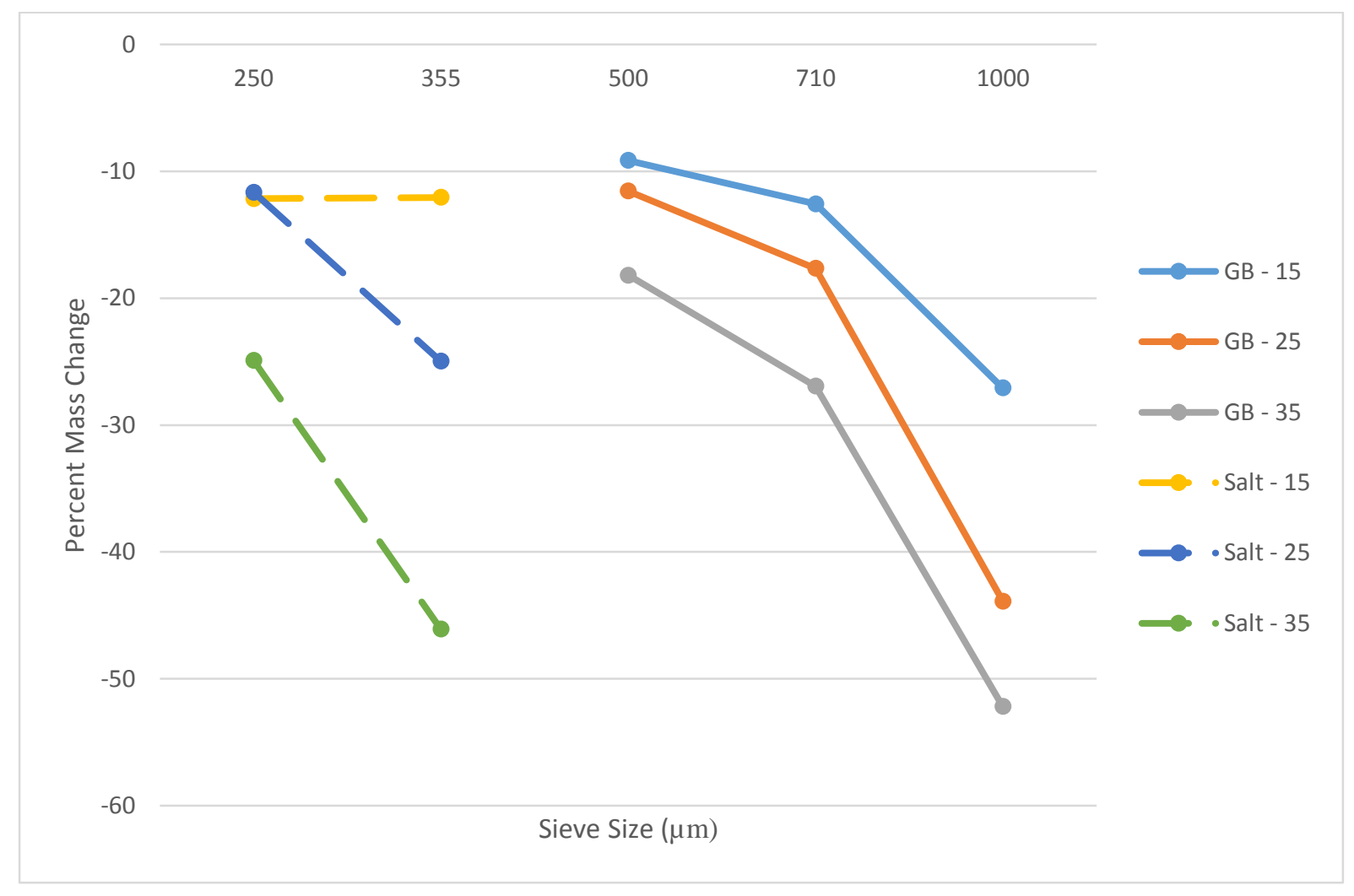

Figure 27 Comparison of Breakage Behaviour with respect to Particle Material, Particle Size and Impact Velocity

A key point of interest is the difference in breakage behaviour between the two materials. Salt appears to have a very distinct threshold, below which little relative breakage occurs. This is shown by the $250-355 \mu \mathrm{m}$ size fraction for salt showing 
almost identical loss in mass for 15 and $25 \mathrm{~m} / \mathrm{s}$, and showing a much greater loss at $35 \mathrm{~m} / \mathrm{s}$. By comparison, the Golden Breadcrumbs display a more progressive breakage characteristic in relation to impact velocity. Furthermore, these results agree well with many previous studies, whereby particle attrition increases with impact velocity $(14,17,38,39)$.

While there are not enough data points in Figure 27 to develop a reliable empirical model, some key trends are evident. Most notably, the apparent higher strength of the smaller particles relative to the larger particles of the same material. This is observed across all tests performed, within the bounds of error.

The critical impact velocity is defined here to be the velocity at which a particle fractures into multiple child particles. This is dependent on factors such as particle material, shape (and therefore impact orientation), size, and for some materials, particle temperature (40). These observations indicate a clear trend that the particle strength increases with decreasing particle size, and support the work of Weibull (41) whereby particles demonstrate a strength distribution. Further analysis is required to quantify this strength distribution, across the entire particle size range and to determine the stochastic particle strength for each material as defined by the critical impact velocity.

\subsection{Importance of Understanding the Product}

The results presented in Sections 4.2 and 4.3 demonstrate the undeniable variability of products in terms of their breakage characteristics. One example of this is the relative breakage observed across the size fractions as the impact velocity is increased. Take the $355 \mu \mathrm{m}$ sieve in Figure 18. As the impact velocity is linearly increased, the percentage mass lost from this fraction exponentially increases 
(doubling each time the impact velocity is increased by $10 \mathrm{~m} / \mathrm{s}$ ). In contrast to this, the percentage mass lost over the same velocity range for the $250 \mu \mathrm{m}$ sieve as shown in Figure 19 doubles when the impact velocity is increased by $20 \mathrm{~m} / \mathrm{s}$ (not accounting for the percentage mass retained at $25 \mathrm{~m} / \mathrm{s}$ ).

To now extend the scope of this analysis, the Golden Breadcrumbs exhibit a doubling in the percentage mass lost on the 1000,710 , and $500 \mu \mathrm{m}$ sieves cross the respective Narrow Size Fraction Tests. Considering that this is an amorphous material, these findings indicate that the material structure dictates sensitivity to impact velocity in attrition. One drawback of these results is that due to the particle size distributions of the materials, comparable particle sizes could not be tested under the Narrow Size Fraction tests, leaving this variable undefined.

\section{Conclusions}

Research into the breakage characteristics of two granulated food products, one crystalline in structure and the other non-crystalline, has been performed. The virgin size distributions were determined, and then the resulting size distributions were tested under three impact velocities: 15,25 and $35 \mathrm{~m} / \mathrm{s}$. Subsequently, three size fractions for golden breadcrumbs and two size fractions for salt were tested under the same conditions as the full size distributions. The results of the latter tests were used to simulate the conditions of the full size distribution breakage characteristics.

The simulated results found good agreement across each of the two materials and the three impact velocities considered. The discrepancies between the experimental and simulated results have been attributed to the combined omission of size fractions larger than those tested, and the migration of the smaller size fractions down the material size distribution. Both discrepancies may be addressed with further testing. 


\section{Future Direction}

The future direction of this work falls primarily into two categories: enhancing the predictive capabilities of this method of analysis, and to enhance understanding of how this analysis may be used in the design and/or optimization process.

Enhancing the predictive capabilities of this method includes looking more holistically at the size distribution and testing the breakage characteristics of every available size fraction. From this, increasing the accuracy of the final size distribution prediction should be achievable.

The application of this analysis would involve obtaining a greater understanding of the process in question, subsequently followed by identifying the undesirable material qualities that result from excess attrition. Determining the ratio of size fractions that exhibit these undesirable qualities will then enable quantification of the process conditions to be avoided.

\section{Abbreviations and Nomenclature}

$[B] \quad$ Breakage matrix

[i] Input matrix

[o] Output matrix

\section{Acknowledgement}

The author would like to express gratitude for the valuable input of others in this programme of research. In particular, Mr Jonathon Larkin for his technical advice, and Mrs Caroline Chapman for her logistical assistance.

The support of BASF SE is gratefully acknowledged for funding this research. 


\section{Appendix}

This appendix presents the partial breakage matrix used to calculate the simulated particle size distribution prior to the prorating process for Golden Breadcrumbs at 35 $\mathrm{m} / \mathrm{s}$ impact velocity as shown below:

$$
[\boldsymbol{B}]=\left[\begin{array}{lllllllllll}
0 & 0.005 & 0.000 & 0.000 & 0 & 0 & 0 & 0 & 0 & 0 & 0 \\
0 & 0.478 & 0.012 & 0.001 & 0 & 0 & 0 & 0 & 0 & 0 & 0 \\
0 & 0.296 & 0.731 & 0.025 & 0 & 0 & 0 & 0 & 0 & 0 & 0 \\
0 & 0.098 & 0.150 & 0.818 & 0 & 0 & 0 & 0 & 0 & 0 & 0 \\
0 & 0.055 & 0.050 & 0.102 & 0 & 0 & 0 & 0 & 0 & 0 & 0 \\
0 & 0.023 & 0.021 & 0.022 & 0 & 0 & 0 & 0 & 0 & 0 & 0 \\
0 & 0.017 & 0.014 & 0.014 & 0 & 0 & 0 & 0 & 0 & 0 & 0 \\
0 & 0.013 & 0.010 & 0.010 & 0 & 0 & 0 & 0 & 0 & 0 & 0 \\
0 & 0.014 & 0.011 & 0.009 & 0 & 0 & 0 & 0 & 0 & 0 & 0 \\
0 & 0.001 & 0.001 & 0.000 & 0 & 0 & 0 & 0 & 0 & 0 & 0 \\
0 & 0.000 & 0.000 & 0.000 & 0 & 0 & 0 & 0 & 0 & 0 & 0
\end{array}\right]
$$

The subsequent prorating process involved adjusting the output particle size distribution to equate to $100 \%$ ensuring that the proportionality of the size distribution was maintained.

The errors are evident in the matrix above whereby the material retained on sieves greater than the feed material size. This was removed and reset to zero as part of the prorating process.

\section{References}

1. Sato S, Shimizu A, Yokomine T. Numerical prediction of erosion for suspension flow duct. Wear [Internet]. 1995 Jul;186-187:203-9. Available from: http://linkinghub.elsevier.com/retrieve/pii/0043164895071377

2. Macchini R, Bradley MS a., Deng T. Influence of particle size, density, particle concentration on bend erosive wear in pneumatic conveyors. Wear [Internet]. Elsevier; 2013 Jun [cited 2015 Oct 1];303(1-2):21-9. Available from: http://inkinghub.elsevier.com/retrieve/pii/S0043164813001695

3. Deng T, Li J, Chaudhry AR, Patel M, Hutchings I, Bradley MSA. Comparison between weight loss of bends in a pneumatic conveyor and erosion rate obtained in a centrifugal erosion tester for the same materials. Wear [Internet]. 2005 Jan [cited 2015 Oct 21];258(1-4):402-11. Available from: 
http://linkinghub.elsevier.com/retrieve/pii/S0043164804001620

4. Burnett AJ. The use of laboratory erosion tests for the prediction of wear in pneumatic conveying bends. University of Greenwich; 1996.

5. Ghadiri M, Zhang Z. Impact attrition of particulate solids. Part 1: A theoretical model of chipping. Chem Eng Sci [Internet]. 2002 Sep [cited 2016 May 17];57(17):3659-69. Available from: http://www.sciencedirect.com/science/article/pii/S0009250902002403

6. Zhang Z, Ghadiri M. Impact attrition of particulate solids. Part 2: Experimental work. Chem Eng Sci [Internet]. 2002 Sep [cited 2016 May 17];57(17):3671-86. Available from: http://www.sciencedirect.com/science/article/pii/S0009250902002415

7. Subero J, Ghadiri M. Breakage patterns of agglomerates. Powder Technol [Internet]. $2001 \quad$ Oct;120(3):232-43. Available from: http://linkinghub.elsevier.com/retrieve/pii/S0032591001002765

8. Antonyuk S, Tomas J, Heinrich S, Mörl L. Breakage behaviour of spherical granulates by compression. Chem Eng Sci [Internet]. 2005 Jul [cited 2016 May 16];60(14):4031-44. Available from: http://www.sciencedirect.com/science/article/pii/S0009250905001442

9. Mishra B., Thornton C. Impact breakage of particle agglomerates. Int J Miner Process [Internet]. 2001 Apr [cited 2016 May 16];61(4):225-39. Available from: http://www.sciencedirect.com/science/article/pii/S030175160000065X

10. Wittel FK, Carmona $\mathrm{H}$ a., Kun F, Herrmann HJ. Mechanisms in impact fragmentation. Int J Fract [Internet]. 2009 Feb 17 [cited 2015 Oct 6];154(12):105-17. Available from: http://link.springer.com/10.1007/s10704-008-9267-6

11. Tong ZB, Yang RY, Yu AB, Adi S, Chan HK. Numerical modelling of the breakage of loose agglomerates of fine particles. Powder Technol [Internet]. 2009 Dec [cited 2016 Apr 20];196(2):213-21. Available from: http://www.sciencedirect.com/science/article/pii/S0032591009004562

12. Deng T, Bingley MS, Bradley MS a., De Silva SR. A comparison of the gas-blast and centrifugal-accelerator erosion testers: The influence of particle dynamics. Wear [Internet]. 2008 Sep [cited 2015 Oct 21];265(7-8):945-55. Available from: http://linkinghub.elsevier.com/retrieve/pii/S0043164808000719

13. Salman A, Gorham D, Verba A. A study of solid particle failure under normal and oblique impact. Wear [Internet]. 1995 [cited 2015 Oct 23];187:92-8. Available from: http://www.sciencedirect.com/science/article/pii/0043164895071407

14. Chapelle P, Abou-Chakra H, Christakis N, Bridle I, Patel MK, Baxter J, et al. Numerical predictions of particle degradation in industrial-scale pneumatic conveyors. Powder Technol [Internet]. 2004 Jun [cited 2015 Oct 1];143144:321-30. Available from: http://linkinghub.elsevier.com/retrieve/pii/S0032591004001822

15. Chapelle P, Abou-Chakra H, Christakis N, Patel M, Abu-Nahar A, Tüzün U, et al. Computational model for prediction of particle degradation during dilutephase pneumatic conveying: the use of a laboratory-scale degradation tester for 
the determination of degradation propensity. Adv Powder Technol [Internet]. Society of Powder Technology Japan; 2004 Jan 1 [cited 2015 Oct 1];15(1):1329. Available from: http://booksandjournals.brillonline.com/content/journals/10.1163/15685520460 740043

16. Frye L, Peukert W. Transfer of fracture mechanical concepts to bulk solids attrition in pneumatic conveying. Int J Miner Process [Internet]. 2004 Dec [cited 2015 Oct 1];74:S279-89. Available from: http://linkinghub.elsevier.com/retrieve/pii/S0301751604000626

17. Bridle I. The Analysis of Particle Degradation in Pneumatic Conveyors Utilizing a Pilot-Sized Test Facility. University of Greenwich; 2000.

18. Kalman H. Attrition control by pneumatic conveying. Powder Technol [Internet]. 1999 Oct;104(3):214-20. Available from: http://inkinghub.elsevier.com/retrieve/pii/S0032591099000972

19. Goder D, Kalman H, Ullmann a. Fatigue characteristics of granular materials. Powder Technol [Internet]. 2002 Jan;122(1):19-25. Available from: http://linkinghub.elsevier.com/retrieve/pii/S0032591001003904

20. Liu L, Kafui KD, Thornton C. Impact breakage of spherical, cuboidal and cylindrical agglomerates. Powder Technol [Internet]. 2010 Apr [cited 2016 May 16];199(2):189-96. Available from: http://www.sciencedirect.com/science/article/pii/S0032591010000264

21. Azimian M, Reiter A, Bart H-J. Computational investigation of impact attrition of particles. Powder Technol [Internet]. 2016 Feb [cited 2016 May 16];289:169-81. Available from: http://www.sciencedirect.com/science/article/pii/S0032591015301984

22. Paluszny A, Tang X, Nejati M, Zimmerman RW. A direct fragmentation method with Weibull function distribution of sizes based on finite- and discrete element simulations. Int J Solids Struct [Internet]. 2016 Feb [cited 2016 May 15];80:3851. Available from: http://www.sciencedirect.com/science/article/pii/S0020768315004357

23. Brosh T, Kalman H, Levy A. Accelerating CFD-DEM simulation of processes with wide particle size distributions. Particuology [Internet]. Chinese Society of Particuology; 2014 Feb [cited 2015 Oct 1];12:113-21. Available from: http://linkinghub.elsevier.com/retrieve/pii/S1674200113001454

24. Hanley KJ, Byrne EP, Cronin K. Probabilistic analysis of particle impact at a pipe bend in pneumatic conveying. Powder Technol [Internet]. 2013 Jan [cited 2016 May 26];233:176-85. Available from: http://www.sciencedirect.com/science/article/pii/S0032591012005736

25. Bika DG, Gentzler M, Michaels JN. Mechanical properties of agglomerates. Powder Technol [Internet]. 2001 Jun [cited 2016 Mar 22];117(1-2):98-112. Available from: http://www.sciencedirect.com/science/article/pii/S0032591001003187

26. Iveson SM, Page NW, Litster JD. The importance of wet-powder dynamic mechanical properties in understanding granulation. Powder Technol [Internet]. 
2003 Feb [cited 2016 Jun 1];130(1-3):97-101. Available from: http://www.sciencedirect.com/science/article/pii/S0032591002002334

27. Ghadiri M, Yuregir KR, Pollock HM, Ross JDJ, Rolfe N. Influence of processing conditions on attrition of $\mathrm{NaCl}$ crystals. Powder Technol [Internet]. $1991 \mathrm{Mar}$ [cited 2016 May 16];65(1-3):311-20. Available from: http://www.sciencedirect.com/science/article/pii/0032591091801950

28. Fu J, Reynolds GK, Adams MJ, Hounslow MJ, Salman AD. An experimental study of the impact breakage of wet granules. Chem Eng Sci [Internet]. 2005 Jul [cited 2016 May 16];60(14):4005-18. Available from: http://www.sciencedirect.com/science/article/pii/S0009250905001429

29. Cleaver JAS, Ghadiri M, Rolfe N. Impact attrition of sodium carbonate monohydrate crystals. Powder Technol [Internet]. 1993 Jul [cited 2016 May 16];76(1):15-22. Available from: http://www.sciencedirect.com/science/article/pii/003259109380036A

30. Aarseth KA. Attrition of Feed Pellets during Pneumatic Conveying: the Influence of Velocity and Bend Radius. Biosyst Eng [Internet]. 2004 Oct [cited 2016 Feb 26];89(2):197-213. Available from: http://www.sciencedirect.com/science/article/pii/S1537511004001217

31. Samimi A, Moreno R, Ghadiri M. Analysis of impact damage of agglomerates: effect of impact angle. Powder Technol [Internet]. 2004 Jun [cited 2016 May 16];143-144:97-109. Available from: http://www.sciencedirect.com/science/article/pii/S0032591004001597

32. Vervoorn PMM, Austin LG. The analysis of repeated breakage events as an equivalent rate process. Powder Technol [Internet]. 1990 Nov [cited 2016 May 27];63(2):141-7. Available from: http://www.sciencedirect.com/science/article/pii/003259109080036X

33. Pathare PB, Baş N, Byrne EP. Attrition of granola as a function of manufacturing process parameters during fluidised bed granulation and subsequent pneumatic conveying. Asia-Pacific J Chem Eng [Internet]. 2013 Sep 10 [cited 2016 May 26];8(5):679-86. Available

from: http://www.scopus.com/inward/record.url?eid=2-s2.084885955609\&partnerID=tZOtx3y1

34. Petersen L, Minkkinen $\mathrm{P}$, Esbensen $\mathrm{KH}$. Representative sampling for reliable data analysis: Theory of Sampling. Chemom Intell Lab Syst [Internet]. 2005 May [cited 2016 May 17];77(1-2):261-77. Available from: http://www.sciencedirect.com/science/article/pii/S0169743904002448

35. van Laarhoven B, Schaafsma SH, Meesters GMH. Toward a desktop attrition tester; validation with dilute phase pneumatic conveying. Chem Eng Sci [Internet]. Elsevier; 2012 May [cited 2015 Oct 1];73:321-8. Available from: http://linkinghub.elsevier.com/retrieve/pii/S0009250911006075

36. Tavares L., King R. Single-particle fracture under impact loading. Int J Miner Process [Internet]. 1998 Jun;54(1):1-28. Available from: http://linkinghub.elsevier.com/retrieve/pii/S0301751698000052

37. Yashima S, Kanda Y, Sano S. Relationships between particle size and fracture 
energy or impact velocity required to fracture as estimated from single particle crushing. Powder Technol [Internet]. 1987 Aug [cited 2016 Feb 15];51(3):27782. Available from: http://www.sciencedirect.com/science/article/pii/003259108780030X

38. Papadopoulos DG, Ghadiri M. Impact breakage of poly-methylmethacrylate (PMMA) extrudates: I. Chipping mechanism. Adv Powder Technol [Internet]. 1996 Jan [cited 2016 Mar 22];7(3):183-97. Available from: http://www.sciencedirect.com/science/article/pii/S0921883108604951

39. Rozenblat $Y$, Grant E, Levy A, Kalman H, Tomas J. Selection and breakage functions of particles under impact loads. Chem Eng Sci [Internet]. $2012 \mathrm{Mar}$ [cited 2015 Oct 1];71:56-66. Available from: http://inkinghub.elsevier.com/retrieve/pii/S0009250911008682

40. Frye L, Peukert W. Progress in the understanding of bulk solids attrition in dilute phase pneumatic conveying. Powder Technol [Internet]. 2004 Jun [cited 2015 Oct 1];143-144:308-20. Available from: http://linkinghub.elsevier.com/retrieve/pii/S0032591004001810

41. Weibull W. A statistical theory of the strength of materials. Vol. 151, Royal Swedish Institute for Engineering Research. 1939. p. 1-45. 